\title{
GEM-DeCan: Improved tumor immune microenvironment profiling through novel gene expression and DNA methylation signatures predicts immunotherapy response
}

Ting $\mathrm{Xie}^{1,2^{\star}}$, Julien Pernet ${ }^{1,2}$, Nina Verstraete ${ }^{1,2}$, Miguel Madrid-Mencía ${ }^{1,2,4}$, Mei-Shiue Kuo ${ }^{3}$, Alexis Hucteau $^{1,2}$, Alexis Coullomb ${ }^{1,2}$, Jacobo Solórzano ${ }^{1,2}$, Olivier Delfour ${ }^{3}$, Francisco Cruzalegui ${ }^{3}$, Vera Pancaldi ${ }^{1,2,4^{*}}$

1 Centre de Recherches en Cancérologie de Toulouse (CRCT), INSERM U1037, Toulouse 31037, France

2 Université Paul Sabatier III, Toulouse 31400, Toulouse, France

3 Translational Medicine, Institut de Recherche Pierre Fabre, Toulouse, France

4 Barcelona Supercomputing Center, Barcelona, 08034, Spain

Keywords: Deconvolution, Tumour microenvironment, DNA methylation, gene expression, EpiDISH, deconRNAseq, quanTIseq, MCP-Counter, Promoter Capture Hi-C, chromatin network

Correspondence to: ting.xie@inserm.fr, vera.pancaldi@inserm.fr 


\section{Abstract}

Quantifying the proportion of the different cell types present in tumor biopsies remains a priority in cancer research. So far, a number of deconvolution methods have emerged for estimating cell composition using reference signatures, either based on gene expression or on DNA methylation from purified cells. These two deconvolution approaches could be complementary to each other, leading to even more performant signatures, in cases where both data types are available. However, the potential relationship between signatures based on gene expression and those based on DNA methylation remains underexplored.

Here we present five new deconvolution signature matrices, based on DNA methylation or RNAseq data, which can estimate the proportion of immune cells and cancer cells in a tumour sample. We test these signature matrices on available datasets for in-silico and in-vitro mixtures, peripheral blood, cancer samples from TCGA, bone marrow from multiple myeloma patients and a single-cell melanoma dataset. Cell proportions estimates based on deconvolution performed using our signature matrices, implemented within the EpiDISH framework, show comparable or better correlation with FACS measurements of immune cell-type abundance and with various estimates of cancer sample purity and composition than existing methods.

Using publicly available data of 3D chromatin structure in haematopoietic cells, we expanded the list of genes to be included in the RNAseq signature matrices by considering the presence of methylated $\mathrm{CpGs}$ in gene promoters or in genomic regions which are in 3D contact with these promoters. Our expanded signature matrices have improved performance compared to our initial RNAseq signature matrix. Finally, we show the value of our signatures in predicting patient response to immune checkpoint inhibitors in three melanoma and one bladder cancer cohorts, based on bulk tumour sample gene expression.

We also provide GEM-DeCan: a snakemake pipeline, able to run an analysis from raw sequencing data to deconvolution based on various gene expression signature matrices, both for bulk RNASeq and DNA methylation data. The code for producing the signature matrices and reproducing all the figures of this paper is available on the GEM-DeCan repository. 


\section{Background}

The tumor microenvironment (TME) is defined as the collection of cells and extracellular matrix that surround cancer cells inside a tumor. It affects tumor development through interactions between the different cells, which impact the probability for cancer cells to escape immune-control, grow and metastasize, and plays an important role in therapy response and resistance [1].

Much of the recent progress in cancer treatment derives from the exploitation and reactivation of immune cells, such as lymphocytes, that infiltrate the TME and fight cancer cells. Despite the great potential of immuno-oncology, there is a considerable difference in efficacy of these therapies across tumor types and patients. It is thus of paramount importance to develop tools to identify the different types of immune cells present in biopsy samples, as this could aid personalised therapy approaches.

More specifically, recent findings about the importance of myeloid cells in hampering the response to immunotherapies make the development of macrophage signature matrices an important goal for immuno-oncology [2]. Traditional immunotherapies rely on the concept that CD8+ T cells, that are normally responsible for killing cancer cells, are already infiltrated in the tumor region, despite being inactivated by their interaction with the cancer cells that disable their cytotoxic activity [3]. The main mechanisms that block the killing of cancer cells were found to rely on the presence of PD-1 and CTLA-4 receptors on the T cell surface, which are inhibited by binding with their specific ligands expressed on cancer cells and antigen-presenting cells respectively [4]. Most current approaches aim to interrupt this interaction using antibodies directed at PD-1 or PDL-1 and CTLA-4, reactivating the killing action of CD8+ $T$ cells against cancer cells. Unfortunately, a high percentage of patients [5] do not seem to respond durably to these treatments and recent findings point to the presence of myeloid cells in the TME of these patients. These myeloid cells can potentially prevent infiltration of CD8+ T cells inside the tumor and even protect cancer cells in some cases [6].

Tumor-associated macrophages (TAMs) can be found in the microenvironment of solid tumors in high numbers. Depending on their phenotypes, these myeloid cells can promote tumor progression, by suppressing antitumor immunity, or directly protecting cancer cells. TAMs are already becoming important treatment targets in cancer, especially in tumors which are not presenting high lymphocyte infiltration $[7,8]$.

Some of these TAMs are differentiated from circulating monocytes upon entering the tumor area while others might be pre-existent residing in the tissue. Macrophages acquire different phenotypes that can be indicatively distributed along a spectrum of polarization going from M1 polarization, in which they promote inflammation, to M2 polarization state, which involves a tumor protective behaviour generated by their role in tissue reconstruction, promoting cancer cell survival [9]. However, these cells are extremely plastic and our knowledge on their behaviour has been mostly generated through in-vitro experiments that probably remain quite distant from the situations they encounter inside tumors $[9,10]$. 
Identifying the presence of different macrophage types in bulk samples has proven particularly challenging due to two main reasons: first, macrophages are not found in the circulating blood, and obtaining them in-vitro requires their stimulation with cytokines that can either produce an M1 or an M2 polarization state in an artificial way, which leaves no guarantee that their phenotype will be comparable to that of TAMs found inside tumors; second, the two macrophage states are very plastic and unstable, while also being quite similar, such that distinguishing between the two states in a bulk dataset is particularly difficult $[9,10]$.

A common approach to identify and quantify the presence of specific cell types in bulk samples after cell separation from tissues or cultures based on their surface markers is Fluorescence Activated Cell Sorting (FACS). However, it is difficult to use FACS to identify cells which are poorly characterised due to a lack of well-defined surface markers, and this technique can become heavy when the samples have multiple cell types and each cell type has to be sorted through multiple markers. Exploiting the wide availability of transcriptomics and methylomics data for bulk samples, multiple algorithms have recently become available to estimate cell type proportions [11] or to estimate tumor purity in complex cellular mixtures [12], a procedure generally referred to as deconvolution Deconvolution methods can be classified as "reference-free" [13,14] or "reference-based" [15] depending on whether a specific signature matrix is used to identify the cell types, or clustering is used to directly infer the different cell types present [16].

DNA methylation (DNAm) profiles are cell-type specific and an excellent alternative to transcriptomes to perform cell-type deconvolution [17]. This is due mostly to the fact that the methylome can be thought of as a record of the cell's past history and is less affected by transient perturbations of the cell's environment [18]. Traditionally, DNAm-based deconvolution was developed for studying different cell types present in blood, specifically in studies regarding the effects of ageing. It was quickly realised that the differences observed in blood across ages could be potentially related to changes in cell-type composition [19].

As for "reference-based" deconvolution methods based on DNAm, the procedure usually involves constructing a signature matrix which is specific and important to the problem of interest. So far, available methods have only used references based on Illumina human 450k or MethylationEPIC (also named 850 k array since it features 850,000 probes along the genome), and are usually estimating absolute fractions referred to the immune cells from blood, such as T cells (CD4+, CD8+), neutrophils (Neu), B cells, natural killer (NK) cells, and monocytes (Mono), but often do not consider other immune cells that are important for cancer immunology like regulatory $T$ cells $\left(T_{\text {reg }}\right)$ and macrophages $(M)$.

In this paper, we exploited a large collection of haematopoietic epigenomes of reference produced by the BLUEPRINT project [20], and published data sets (Additional file 1) to establish a series of novel signature matrices for a gene expression and methylation based deconvolution approach. In addition to novel signatures for expression or methylation based deconvolution of immune cell types in blood (BPmet, BPRNA), we generated specific signature matrices to quantify the proportion of cancer cells at the same time as specific immune cell types infiltrated in tumours (BPmetCan, BPRNACan). 
We further hypothesized that genes that are not passing the filter of inclusion in the gene expression-based signature matrix, but are associated with $\mathrm{CpGs}$ that are in the methylation signature matrix, should also be included in the gene-expression deconvolution signature matrix. We exploited the available chromatin structure in haematopoietic cells [21] to identify genes whose expression might be impacted by $\mathrm{CpGs}$ in the DNAm signature matrix, locally or through $3 \mathrm{D}$ contacts, and were able to show that our expression-based signature matrix is improved when including these genes. We further evaluate the performance of the different signatures and approaches based on their potential as biomarkers to predict response to anti-PD1 treatment.

Finally, we provide the GEM-DeCan pipeline to allow for gene expression and DNA methylation data processing, in order to run different deconvolution methods with multiple provided signature matrices. Along with the pipeline, a script to guide users into the generation of their own signature matrix is supplied.

\section{Methods}

\section{Collection of WGBS data}

To build the Blueprint signature matrix, we collected 52 samples generated from whole-genome bisulfite sequencing (WGBS) of 10 purified blood-derived immune cells on GRCh38 (Homo sapiens genome) (Additional file 1: Table S1). Bigwig files including methylation signal and coverage of methylation signal were downloaded from Blueprint epigenome level 3 data (http://www.blueprint-epigenome.eu). In addition, we also downloaded 7 cancer datasets, 4 WB and 11 normal tissues (Additional file 1: Table S2) via Gene Expression Omnibus (GEO, https://www.ncbi.nlm.nih.gov/geo/) and Brinkman et al. [22].

\section{WGBS data processing}

The files were parsed into $R$ data structures, we then discarded bases that have coverage below 10X [23] and also have more than 99.9th percentile of coverage in each sample. Methylation signal $\left(\mathrm{WGBS}_{\beta}\right)$ was calculated with the following formula:

$$
\text { WGBS }_{\beta}=\text { methylatedCounts / (methylatedCounts + unmethylatedCounts) }
$$

The hg19 coordinates of WGBS from GEO were converted to GRCh38 with the liftOver R package. To map the common methylated cytosines from GEO and Blueprint datasets by genomic position to the Illumina 850K Methylation Array CpG sites, we used the Infinium MethylationEPIC v1.0 B5 Manifest (https://support.illumina.com/downloads). After the coordinate transformation, the missing values on the beta matrix were imputed with impute $R$ package [24] using default parameters to generate the final beta matrix without missing values. 
A beta-value matrix was generated from the WGBS dataset, including 409,103 CpGs (48\% overlap with EPIC's 850k CpGs) measured across a total 107 samples (Normal=17, WB=4, Cancer=34, Immune=52).

\section{Deconvolution Blueprint (BP) signature matrices generation procedure}

The signature matrices were established through the following steps:

1. The limma [25] $R$ package was used to determine differentially methylated CpGs (DM-CpGs) or differentially expressed genes (DEGs) for all pairwise comparisons between cell types: for GE signature matrices, the voom function was used to remove heteroscedasticity for RNA-seq data using log2(TPM + 1).

2. We preselected significant candidate DM-CpGs/DEGs for the signature matrices using Benjamini-Hochberg corrected $p$-values. For GE signature matrices, a false discovery rate (FDR) cutoff of 0.05 was selected, while for the DM-CpGs, 2 different FDR values were selected (Additional file 2: Method S1) to generate their signature matrices:

a. FDR $<0.05$ was used to generate BPmet in blood.

b. FDR $<1 \mathrm{e}-05$ was used to generate BPmetCan.

3. The significant candidate genes/CpGs that were obtained from the previous step (point 2) were selected according to specific thresholds based on their absolute logFC for pairwise comparisons (Additional file 2: Method S1). We filtered the DEGs with 2 different abs( $\log F C)$ cutoffs to obtain our GE signature matrices:

a. $\operatorname{abs}(\log \mathrm{FC})>2.5$ was used to generate the BPRNA signature matrix.

b. $\operatorname{abs}(\log F C)>2$ was used to generate BPRNACan immune cell signature matrices, while abs $(\log F C)>3$ was used to obtain BPRNACan cancer cell signatures .

We then sorted the genes obtained from point 3 and ranked them by decreasing fold change (to obtain positive variable genes). The number of genes to be included in each signature was chosen depending on their logFC density distributions (Additional file 2: Method S1).

\section{Generation of the DNA methylation signature matrix (BPmet)}

We started from WGBS public datasets from the Blueprint project to generate a methylation based signature matrix (BPmet), which we used for immune cell decomposition in blood from healthy donors. Datasets for purified immune cells of 6 types were considered: CD4 $(\mathrm{N}=8)$, CD8 $(N=8)$, B cells $(N=9)$, Monocytes $(N=4)$, NK $(N=2)$ and Neutrophils $(N=7)$ [20]. The signature matrix includes only $\mathrm{CpGs}$ with false discovery rate $(\mathrm{FDR})<0.05$, absolute log fold change $(\mathrm{abs}(\mathrm{Ifc}))>0.2$ and the top $100 \mathrm{CpGs}$ (Additional file 3: Table S1).

\section{Generation of the enhanced DNA methylation signature matrix for cancer samples (BPmetCan)}

To identify cancer cells, normal cells and immune cells in tumour samples, we used firstly a set of Normal (tissues), Whole Blood (WB) and cancer (solid tumours) WGBS samples 
(Normal: $\mathrm{N}=17, \mathrm{WB}: \mathrm{N}=4$, Cancer: $\mathrm{N}=34$ ) via GEO and Brinkman et al. [22] to generate a signature matrix that can recognize the three groups of cells. To identify the CpGs we needed to include in this signature matrix, we chose CpGs that were highly methylated in cancer cells compared to Normal as well as WB samples with cutoffs at FDR $<0.05$, Ifc $>$ 0.5 , selecting a maximum of $100 \mathrm{CpG}$ for each pairwise comparison between the 3 cell type group.

In the tumor microenvironment, three states of macrophages can be found (M0, M1, M2) [26] as well as one specific type of T cell (Treg) [27]. For this reason, we extended the BPmet signature matrix with those 4 new immune cell types to generate a new BPmet signature matrix following the signature matrix process section with FDR threshold of $1 \mathrm{e}-05$, Ifc $>0.3$ and with a maximum of $300 \mathrm{CpGs}$ for each pairwise comparison.

To build the final BPmetCan signature matrix, which can identify cancer cells and also specific immune types, we merged the above described signature matrix for cancer/normal/blood cells with the BPmet signature matrix extended with macrophages and Tregs. The final significant $\mathrm{CpGs}$ are the union of $\mathrm{CpGs}$ presented in those two signature matrices, respectively, whereas the profiles of $\mathrm{CpGs}$ in each cell type were calculated through the median of methylated values over all samples belonging to that cell type. The BPmetCan signature matrix includes 1896 CpGs (Additional file 3: Table S2).

\section{RNAseq data processing}

The TCGA expression data normalized as Fragments Per Kilobase of transcript per Million (FPKM) were taken from public datasets [28]. The transcripts per millions (TPM) expression datasets for WB were downloaded from GTEx portal (https://gtexportal.org/home/). 9 purified blood-derived immune cells TPM expression datasets on GRCh38 (Additional file 1: Table S3) were collected from the Blueprint project portal [20]. The expression value FPKM was first converted to TPM using the following formula:

$$
\mathrm{TPM}_{\mathrm{i}}=\left(\mathrm{FPKM}_{\mathrm{i}} / \sum \mathrm{FPKM}_{\mathrm{j}}\right) * 10^{6}
$$

The final expression value for each gene $e_{i}$ in the sample $e_{j}$ was normalized from TPM with the following formula:

$$
\mathrm{TPM}_{\mathrm{ij}}=\mathrm{TPM}_{\mathrm{ij}}{ }^{*} 10^{6} / \sum_{\mathrm{t}} \mathrm{TPM}_{\mathrm{tj}}
$$

\section{Generation of the BPRNA signature matrix}

We generated the Blueprint RNAseq based signature matrix (BPRNA) using the same approach and the same 6 immune cell types as for the BPmet signature matrix. We selected the signature genes as the top 200 genes (ranked by decreasing Ifc), among those which have false discovery rate (FDR) $<0.05$ and Ifc $>2.5$ (Additional file 3: Table S3).

\section{Generation of the CCLE_TIL10 signature matrix}

To develop this signature matrix we started from the TIL10 (170 genes) one proposed in the quanTIseq method[29], which includes 10 immune cell types (B cells, M1 and M2 
macrophages, monocytes (Mono), neutrophils (Neu), natural killer (NK) cells, non-regulatory CD4+ T cells, CD8+ T cells, Treg cells, and myeloid dendritic cells (DC)).

FASTQ files of all samples used for generating the TIL10 signature matrix (170 genes [29]) were downloaded, preprocessed and gene expression was quantified as described in [29]. An expression matrix, for 10 immune cell types, was constructed, consisting of 19,423 genes and 51 samples. We then constructed a cancer signature matrix ("CCLE") by considering differential expression between cancer cell line samples from CCLE (eliminating blood cancer cell lines) [30] and healthy tissues and blood samples from GTEx [31]. Briefly, we used a bootstrap approach by which 50 samples were randomly taken from each of the three datasets to construct the complete dataset (150 samples in total) for the analysis of differential expression by limma. The mean-variance relationship was modeled with the voom function and the Benjamini-Hochberg method was used for multiple hypothesis testing. IfC $>2.5$ and FDR $<0.005$ were used to select differentially expressed genes. Only genes which are highly expressed in cancer cells compared to normal tissues and compared to blood samples were selected as "UP" genes. This procedure was repeated 30 times and only the UP genes which are present in each iteration are selected as cancer cell specific genes (Fig. 2b). The expression profile of cancer cells was computed as the median of the expression values over all samples for all UP genes in the CCLE dataset, resulting in the CCLE signature matrix (138 genes).

To build the combined CCLE_TIL10 signature matrix, we simply considered the union of genes from the TIL10 and CCLE signature matrices (Fig. 2b). The expression profiles in the matrix were computed as the median of the expression values over all samples belonging to each cell type (Additional file 3: Table S4).

\section{Generation of the BPRNACan signature matrix}

We generated first an extended BPRNA signature matrix, based on Blueprint expression data [20], selecting samples for CD4 $(N=12)$, CD8 $(N=3)$, B cells $(N=5)$, Monocytes $(N=7)$, $\mathrm{M0}(\mathrm{N}=4), \mathrm{M} 1(\mathrm{~N}=4), \mathrm{M} 2(\mathrm{~N}=5), \mathrm{NK}(\mathrm{N}=2)$ and Neutrophils $(\mathrm{N}=10)$ and filtering out Treg cells due to low number of samples $(\mathrm{N}=1)$. We then selected genes with an FDR $<0.05$, and IfC $>$ 2 , including the 150 genes with highest Ifc to identify differentially expressed genes, following the procedure to create the signature matrix. Second, we built a cancer signature matrix using normal and cancer samples collected from TCGA for different cancer types with normal adjacent tissue and WB from GTEx, using FDR < 0.05, Ifc > 3, including the top 100 genes with highest Ifc. Samples from TCGA were from 18 cancer types: Bladder, Cholangiocarcinoma, Thyroid carcinoma, Liver hepatocellular carcinoma, Colon adenocarcinoma, Kidney Chromophobe, Kidney Renal Clear Cell Carcinoma, Kidney renal papillary cell carcinoma, Lung Squamous Cell Carcinoma, Lung Adenocarcinoma, Stomach adenocarcinoma, Cervical squamous cell carcinoma and endocervical adenocarcinoma, Uterine Corpus Endometrial Carcinoma, Head-Neck Squamous Cell Carcinoma, Breast invasive carcinoma, Rectum adenocarcinoma, Esophageal carcinoma. The final BPRNACan signature matrix was generated by merging the two signature matrices described above, and includes 1403 genes (Additional file 3: Table S5). 
Generation of gene expression signature matrices expanded according to the methylation signature matrix (BPRNACanProMet) and 3D chromatin contact maps (BPRNACan3DProMet)

Our BPRNACan signature matrix contains 1403 genes which we call sig genes, whereas the $1896 \mathrm{CpGs}$ from the BPMetCan signature matrix are denoted as sig CpGs. To take into account the potential involvement of genes that are important for each cell type, as evidenced by methylation, but not sufficiently differentially expressed to be sig genes, we created a set of expanded gene expression signature matrices.

We considered 3D chromatin contact networks for all immune cells included in Javierre et al. [21], detected by the Promoter Capture Hi-C technique and filtered using CHiCAGO [32]. First, we combined BPRNACan sig genes with the genes that have a BPMetCan sig CpG in their promoter (according to promoter definitions based on promoter capture libraries in [21]), leading to the "BPRNACanProMet" signature matrix (Additional file 3: Table S6). Additionally, we generated the "BPRNACan3DProMet" signature matrix by adding the genes that have a sig $\mathrm{CpG}$ in their promoter and having a 3D contact with a fragment containing a sig CpG, (Additional file 3: Table S7). Finally we constructed a further expanded signature matrix adding genes whose promoters only have a $3 \mathrm{D}$ contact with a fragment containing a sig $C p G$, leading to the "BPRNACan3DMet" signature matrix.

\section{RNAseq processing and deconvolution pipeline}

In order to test the different signature matrices we present in this paper, we provide an RNAseq analysis pipeline, built with snakemake [45] and conda [46]. It allows the user to choose from various tools and options (Fig. 1). The pipeline can start from raw Illumina sequencing data (.bcl format) or from already processed and normalized TPM data and runs a selection of tools performing deconvolution with the methods used in this paper (quanTIseq, MCPCounter, deconRNASeq, and EpiDISH) and all signature matrices mentioned in the paper. It is freely available on the GEM-DeCan repository.

\section{Validation datasets}

\section{Whole blood methylation datasets}

For validating and assessing the performance of the BPmet signature matrix, we used two independent public datasets (Additional file 2: Table S1): 100 WB samples from the Grady Trauma Project (GSE132203) profiled using IlluminaHumanMethylationEPIC and another 6 WB samples using the 450k methylation array from Koestler et al. (GSE77797) [33]. Flow-cytometry estimates of the proportion of blood cell types were available for the two WB datasets. The estimated fraction of cells obtained by deconvolution using the EpiDISH (RPC: robust partial correlation) method [34] was compared to the flow-cytometry estimated proportions using Pearson Correlation.

\section{Methylation datasets from Peripheral Blood Mononuclear Cells (PBMC)}

To test our BPRNA deconvolution signature matrix on whole blood, we used 13 PBMC samples with corresponding flow cytometry data [35] (Additional file 2: Table S1). 


\section{In-silico mixtures}

To test the BPRNACan signature matrix, we used a simulated RNA-seq dataset from quanTIseq [11], consisting of 1700 samples created by in-silico mixing of reads from immune-cells and cancer cell lines in different proportions, simulating different tumor purity (0 to 100\%) (Additional file 2: Table S1).

\section{Melanoma cancer samples}

To assess the performance of our GE signature matrices, we used 4 metastatic melanoma lymph nodes from Racle et al. (GSE93722) [36] and another 19 primary tumor samples from non-metastatic patients from Tirosh et al. (GSE72056) [37] (Additional file 2: Table S1).

\section{Cancer samples with methylation and gene expression profiles}

To test the BPmetCan and BPRNACan signature matrices, 495 methylation profiles from LUAD samples in TCGA were downloaded in level 3 (beta-value, 450k Illumina array) as well as their corresponding gene expression profiles measured by RNAseq (RNASeq2GeneNorm) using the RTCGAToolbox [38] R package. Finally, we also considered 59 RNA-seq datasets from multiple myeloma bone marrow samples after removal of cancer cells [39] (Additional file 2: Table S1).

\section{Estimating accuracy in cancer cell proportion deconvolution}

To estimate the proportion of cancer cells inside tumor samples, different methods have been proposed. For TCGA samples, estimates based on the ABSOLUTE, ESTIMATE, LUMP and IHC methods were available in [12] as described in Additional file 2: Table S2.

\section{Comparison between proportions estimated by deconvolution and other methods}

We used Pearson's correlation coefficients to compare our estimates of deconvolved proportions to either FACS data or alternatively estimated proportions (Additional file 2: Table S2).

\section{Regression models to predict response to immunotherapy}

Three public melanoma datasets and one bladder cancer dataset with response to anti-PD1 [40-43] were considered. ElasticNet [44] penalized logistic regression models were run using the results from different deconvolution methods and signature matrices as features.

For each combination of signature matrix and deconvolution method, 5 models were trained, including 4 models trained by leave-one-dataset-out (lodo) and one model trained by 5 -fold cross-validation (standard CV). The training includes a hyperparameter search for the 11 ratio and penalty strength. For the lodo training this search is performed by 5 -fold $\mathrm{CV}$ on training datasets, and models are evaluated on the remaining test dataset. For the standard $\mathrm{CV}$, one fourth of samples is kept as a hold-out test set, and the hyperparameter search is performed by 5 -fold $\mathrm{CV}$ on the remaining samples, the model is then evaluated on the hold-out test set. 


\section{Results}

\section{BPmet: A Novel DNA methylation-based signature matrix for immune cell deconvolution}

We exploited the WGBS methylation datasets that were produced for bulk samples of purified cells as part of the Blueprint project [47] to generate a signature matrix of 502 CpGs which allows us to identify 6 major immune cell types in blood (see methods, Fig. 2a and Additional File 1: Table S1). We named this signature matrix BPmet (Additional file 3: Table S1). To test this newly generated signature matrix, we performed deconvolution with the EpiDISH R package [34], using our BPmet signature matrix, and choosing the RPC method as well as other available methods (Additional file 2: Table S2). We then proceeded to test our BPmet new signature matrix on various datasets (Additional file 2: Table S1).

\section{Testing the BPmet signature matrix on peripheral blood samples}

To evaluate the performance of the BPmet signature matrix using the EpiDISH method, we first applied it to an independent publicly available Illumina EPIC array (850k CpGs) dataset choosing 100 whole blood samples from healthy donors with the true cell composition verified by FACS (gold standard) (GSE132203, Grady Trauma Project). We observed extremely high Pearson correlations between the estimated cell compositions and FACS fractions for all included samples (Pearson's $R=0.993, p<2.2 e-16$ ) and each cell subtype (Fig. 3a and Additional file 2: Figure S1a).

We then compared the results from using the BPmet signature matrix in these 100 whole blood samples from the previously mentioned gold standard dataset (GSE132203) to the results using other signature matrices. The signature matrices we compared were the MethyICIBERSORT signature matrix [48], and the default signature matrix for the EpiDISH method [34], based on DNAse hypersensitivity sites (DHS), which are highly cell-type specific regions of open chromatin [49]. Altogether, correlations between the different signature matrix estimates and the FACS fractions were similar for EpiDISH-DHS and BPmet, while MethyICIBERSORT had a worse performance. Only NK cells estimate was worse correlated with BPmet compared to EpiDISH-DHS and MethyICIBERSORT, probably due to the few samples included for this cell type in creating our signature matrix $(\mathrm{NK}: \mathrm{n}=2)$ (Additional file 2: Figure S1a-c)

Since the MethyICIBERSORT and EpiDISH-DHS signature matrices were generated starting from the Illumina 450k platform, we tested them on another set of 6 whole blood samples analysed by the same technology and compared results to the flow cytometry measurements provided for that dataset [33]. We were able to obtain better correlations (Pearson's $\mathrm{R}=0.93, \mathrm{P}<2.2 \mathrm{e}-16$ ) for all cell types confounded compared to either methyICIBERSORT or EpiDISH-DHS. We obtained correlations above 0.94 for each cell subtype except for NK cells (Fig. 3b and Additional file 2: Figure S2). We thus conclude that our BPmet signature matrix can correctly capture cell type composition as well or better than other available methods in whole blood. 


\section{Extending the BPmet signature matrix to estimate cancer and immune cell proportion in tumor samples}

Confident of the satisfying performance of the BPmet signature matrix in identifying cell type proportions in whole blood samples, we turned towards applying deconvolution to cancer tissues, extending the BPmet signature matrix to also estimate the proportion of cancer cells. To expand our BPmet signature matrix to include immune cell types in the TME and also cancer and normal cells of different types, we combined data from the previously used 52 immune samples with 17 normal and 34 cancer samples (see Methods for details). This signature matrix, called BPmetCan (see Methods, Fig. 2a and Additional file 3: Table S2), allowed us to estimate the proportion of cancer cells as well as of 10 immune cell types in bulk tumor samples.

To test the performance of BPmetCan in a realistic scenario, we used it to estimate the proportion of cancer cells in 495 Lung Adenocarcinoma (LUAD) DNA methylation datasets from TCGA. Firstly, we retrieved publicly available estimates of the proportion of cancer cells in these samples (purity) produced using the ABSOLUTE method [50], which involves analysing somatic DNA alterations to estimate ploidy and hence tumor purity. When comparing purity estimates using BPMetCan with ABSOLUTE results, we obtained a Pearson correlation of $\mathrm{R}=0.79$ and $\mathrm{P}<2.2 \mathrm{e}-16$, proving that our signature matrix is able to identify the proportion of cancer cells in a tumor sample (Fig. 4a). We then compared the results obtained with BPmetCan to other previously published purity estimation methods from ref $[12,48]$. These include a method based on gene expression of immune and stromal genes (ESTIMATE [51]), a method based on CpGs that are unmethylated in immune cells (LUMP [12]), a methylation based deconvolution approach (MethyICIBERSORT [48]) and a method that estimates purity based on images of haematoxylin and eosin stain slides (IHC [12]).

We found that BPmetCan displayed a higher correlation with ABSOLUTE than either ESTIMATE or IHC, and a very similar one to MethyICIBERSORT and LUMP in TCGA-LUAD (Fig. 4a). In order to further verify whether our signature matrix is accurate in calculating the proportion of tumor purity in other cancer types, we also applied it to breast cancer samples in TCGA (TCGA-BRAC). Once again, we observed a high correlation of estimated tumor purity between BPmetCan and ABSOLUTE, and our method had the second best concordance with ABSOLUTE after MethyICIBERSORT (Additional file 2: Figure S3).

In order to assess our ability to determine the proportions of other immune cell types in the sample, the estimated fraction of immune cells using BPmetCan or MethyICIBERSORT signature matrix was compared to quantification of lymphocytes, macrophages and neutrophils estimated from H\&E images also in the same samples from TCGA-LUAD [52]. We observed that BPmetCan and MethyICIBERSORT have very similar performances, as measured by the correlation of deconvolved immune cell types compositions with H\&E estimates (Fig. 4b). Investigating macrophages (Monocytes/Macrophages lineage) showed that the prediction of macrophage abundance derived from MethyICIBERSORT was more accurate than that derived using the BPmetCan signature matrix $(R=0.48$ against $R=0.33$ ) 
(Fig. 4b). Nevertheless, it appears that the MethyICIBERSORT reference profiles do not capture all macrophages (some samples are on the $\mathrm{x}$-axis indicating no estimated abundance while non-zero values are clearly detected by H\&E images). This could be due to the classification of macrophages by the MethyICIBERSORT signature matrix that only includes monocytes (CD14+ cells), whereas our signature matrix can potentially classify monocytes, M0, M1 and M2 separately (all these types were merged for making this comparison).

\section{BPRNA: a signature matrix for immune cell deconvolution in blood}

We started by developing a novel immune cell type deconvolution signature matrix based on RNAseq expression data from primary samples for 6 immune cell types (see BPmet part, including CD4 T cells, CD8 T cells, Monocytes, B cells, NK cells and Neutrophils) in blood [20], which we called BPRNA (see methods, Fig. 2a and Additional file 3: Table S3). We then proceeded to test the BPRNA signature matrix using two deconvolution methods for which signature matrices can be specified by the user (EpiDISH [34] and deconRNAseq [53]) on peripheral blood mononuclear cell (PBMC) samples (Additional file 2: Table S1).

\section{Testing the BPRNA signature matrix on PBMC samples}

We tested our BPRNA signature matrix using PBMC mixtures where both RNA-seq and flow cytometry data was available [34]. We observe lower accuracy of estimated total cell fractions for this dataset, especially for NK and CD8 cells (Fig 5a). This is probably due to the limited number of samples available for $\mathrm{NK}(\mathrm{N}=2)$ and to a possible discrepancy between the CD8 activation state in these PBMC samples and the ones used in the signature matrix (which exclude naive CD8 cells).

Multiple gene expression reference-based methods have demonstrated a high accuracy in estimating cell proportions in blood and immune infiltrates from bulk RNA-seq data, amongst which we chose two for comparison to estimates produced by BPRNA: we first compared to two methods that come with their own signature: MCP-counter [54], which is a scoring method based on marker genes, and quanTlseq [29], which is based on constrained least squares regression and can estimate immune cell fractions and fractions of unknown cells with high accuracy. Finally, we tested the deconRNAseq [53] method, using different signature matrices, to compare its performance with EpiDISH. The results of running deconvolution with all different methods and signature matrices compared to FACS estimates in PBMC are summarised in Figure 5b.

Considering all cell types together or sub-cell types, we observed the combination of BPRNA with EpiDISH compared very favorably relative to the combination of BPRNA with deconRNAseq, which overall showed the weakest performance (Fig 5b). However, we note that MCP-counter or quanTIseq outperformed the combination of BPRNA with EpiDISH in several sub-cell types (CD8 T cells, Monocytes, B cells, NK cells, and Neutrophils). 


\section{BPRNACan and CCLE_TIL10: new immune-cell and cancer gene expression signature matrices}

To exploit the wide availability of expression data for cancer samples, we generated two gene expression-based signature matrices to identify immune and cancer cells. Similar to what was done for the methylation based signature matrix, we extended the BPRNA signature matrix to detect cancer cells as well as specific immune types.

We considered two ways of designing a signature matrix that would estimate tumor purity based on bulk RNAseq expression data. In the first case, we constructed a signature matrix named CCLE_TIL10 (see methods and Additional file 3: Table S4) based on RNAseq data for over a thousand cancer cell lines [30] and a large number of healthy tissue and blood RNAseq samples from the GTEx [31].

Aware of the difference between cancer cell lines and cancer cells, we also developed a signature matrix for detecting cancer and specific immune cells starting from expression data in cancer, adjacent normal tissues and immune cells. To this end, similarly to what was done in the case of DNA methylation, we looked for samples with RNAseq data for tumor and non-tumor tissues and whole blood, which are readily available through the TCGA and GTEx, and integrated it with the BPRNA immune cell signature matrix, which was based on 9 immune cell types [20]. This new signature matrix, which we called BPRNACan consists of 1403 genes (see Methods, Fig. 2a and Additional file3: Table S5). We used the CCLE_TIL10 and BPRNACan signature matrices with the EpiDISH method to estimate cell composition in different samples with gene expression datasets (Additional file 2: Table S1).

\section{Validation of the CCLE_TIL10 and BPRNACan signature matrices on in-silico and in-vivo tumor samples}

As a first step to validate our method on samples containing cancer cells, we considered an in-silico simulated cancer RNAseq dataset, composed of reads from purified samples from 10 immune cells (B-cells, NK-cells, CD4+ T-cells, CD8+ T-cells, Monocytes, NK, Neutrophils, M1, M2 and Dendritic cells), which were added to reads from a sample of MCF10 cancer cell lines in different proportions [29]. The results of using the CCLE_TIL10 signature matrix on this in-silico mixture are in excellent agreement with true mRNA proportions (Pearson $\mathrm{R}>$ 0.9), for 11 cell types (Additional file 2: Figure S4a), as expected, since the TIL10 signature matrix was derived from this data and CCLE_TIL10 signature matrix is an extension of it.

The proportions estimated using our newly developed BPRNACan signature matrix (with the EpiDISH method) were also in very good agreement with the true mRNA proportions (Pearson $\mathrm{R}=0.96$ for cancer cells and Pearson $\mathrm{R}>0.74$ for other immune cells fractions, Additional file 2: Figure S4b), except for macrophages $M 2(R=0.35)$. This lower performance in the detection of M2 macrophages could be due to BPRNACan missing M2s in some samples, suggesting that the signature matrix potentially does not capture all M2 phenotypes that are present. Our signature matrix might also have similar issues with M1 
macrophages, as we can clearly see two groups of samples, one of which has estimated M1 proportions quite discordant from the true cell fractions (Additional file 2: Figure S4b).

We then investigated whether these deconvolution signature matrices would be able to estimate tumor purity from real biological samples, namely tissue samples from TCGA. We therefore analysed the results obtained with the 2 signature matrices on TCGA samples (Additional File 2, Figure S5). We first compared the estimation of cancer cell purity in the samples to ABSOLUTE, ESTIMATE, LUMP and IHC results, as was done for the methylation analysis. We found that the tumor purity estimates derived using BPRNACan were better than those derived from CCLE_TIL10 on the TCGA-LUAD dataset, with correlations with the ESTIMATE method reaching Pearson's $\mathrm{R}=0.72$ (Additional File 2, Figure S5b).

As far as different cell types are concerned, we again compared our deconvolved proportions to estimations based on H\&E images [52] (Additional file 2: Table S1). Interestingly, once again, the BPRNACan signature matrix performed better than CCLE_TIL10 on biological samples (Fig. 6a). However, we observed the correlation for neutrophils was lower than expected, probably due to the presence of neutrophils inside tumors with a specific phenotype not captured by our signature matrix. In order to further test our signature matrices using a different method from $\mathrm{EpiDISH}$, we again compared results to the ones obtained by deconRNAseq with our two signature matrices, MCP-counter and quanTIseq. We observed that the deconRNAseq method, using both CCLE_TIL10 or BPRNACan signature matrices, produced estimates in weak agreement with H\&E for all immune cell types. On the contrary, MCP-counter and quanTlseq estimates showed high correlations with H\&E estimates for lymphocytes, but strikingly low correlations for neutrophils (Fig. 6b and Additional file2: Figure S6). In summary, we observed that none of the methods achieved a high correlation with H\&E for neutrophil proportions. However, in terms of accuracy, BPRNACan with EpiDISH emerged as the best performing method for this dataset (Fig. 6b and Additional file2: Figure S6b).

These analyses demonstrated that since the CCLE_TIL10 signature matrix was generated based on in-silico samples, it is accurate for in-vitro and in-silico mixtures, whereas BPRNACan performs better on biological samples, in which cells are in a biologically relevant context, including the exchange of signals between them. Thus we next tested our two signature matrices on additional in-vivo cancer samples.

To further benchmark the methods, we used a series of 59 multiple myeloma patient TME samples, generated by removal of cancer cells from bone marrow samples [39]. For this dataset FACS analysis was performed to quantify NK, CD4 and CD8 T cells so we could compare our estimations for these cell types from the different methods to FACS results. Although only 3 immune cell types had been assayed in this study, BPRNACan produced higher correlations with FACS for CD4 T and NK cells, and lower correlations for CD8 T cells compared to quanTIseq (Fig. 6c), while for all three cell types it gives higher correlations than MCP-counter (Fig. 6c). 
Finally, we examined a single-cell RNAseq dataset including 19 reconstructed primary melanoma non metastatic samples [37] and aggregated reads to produce an in-silico bulk sample for each patient to compare deconvolved proportions to the corresponding cell fractions estimated by counting single cells of each type from Racle et al. [36] (CD8 T cells, macrophages, B cells and NK cells). We ran EpiDISH deconvolution using the BPRNACan signature matrix and measured high correlations with the cell-type proportions estimated in the publication (Pearson's $\mathrm{R}>0.7$ for all 4 cell types, Fig. $6 \mathbf{d}$ and Additional file 2: Figure S6c). Moreover, we also explored four metastatic melanoma patients with available flow cytometry data (for CD4 T cells, CD8 T cells, B cells, NK cells, melanoma cells) provided in the same publication [36]. Upon comparing the cell proportion estimates derived using BPRNACan with estimates derived from MCP-counter and quanTIseq, we observed that BPRNACan has the most robust performance for cancer cells, CD4 and CD8. In addition, we once again observed the combination of BPRNACan with EpiDISH performed better than the combination of BPRNACan with deconRNAseq (Additional file 2: Figure S6d).

\section{Gene expression and methylation based cell type signatures are significantly associated}

In this part we investigated what could be the relation between the genes that are included in the BPRNACan signature matrix gene set (1403 sig genes) and the CpGs from the BPMetCan signature matrix (1896 sig CpGs), assuming that comparing both methylome and transcriptome of purified cells would yield similar genes as important in defining the cell type. We calculated the number of sig genes that are associated with the sig CpGs. To associate CpGs to genes we first used Illumina annotation, which provides gene associations for each CpG on their array, based on genomic proximity between the gene and the CpG. Out of 169 genes associated with sig CpGs, 24 were included in the 1403 sig genes (Fisher's exact test p-value=1e-5) (Additional File 2: Table S4a).

\section{Combining the methylation and expression based signature matrices}

Inspired by the availability of 3D chromatin contact maps for haematopoietic cells produced by the Blueprint project [21] and by known relations between 3D conformation, methylation and expression levels [55], we decided to re-evaluate CpGs annotation to genes and consider 3D chromatin contacts between these $\mathrm{CpGs}$ and gene promoters, as identified by Promoter-Capture Hi-C (PCHi-C). Briefly, this method involves a step of hybridization to a promoter library during a traditional $\mathrm{Hi}-\mathrm{C}$ experiment, resulting in chromatin contact maps that contain either contacts amongst promoters or between a promoter and a regulatory region (denoted as OE for Other end or PIR for Promoter Interacting Region), where chromatin fragments have a median size of $5 \mathrm{~kb}$ [56]. The total PCHi-C network for haematopoietic cells consists of 249,511 chromatin fragments, of which 20,582 are promoter fragments (including 1127 fragments of sig gene promoters) and 228,929 other ends, (including 1131 fragments containing sig CpGs) (Additional file 2: Table S3).

We first mapped sig CpGs to their corresponding gene promoters, according to the definition of promoters used in the PCHi-C datasets. We found 645 genes that have sig $\mathrm{CpGs}$ in their promoter, of which 52 were already identified in sig genes and 314 were not in sig genes and 
had expression profiles in our reference datasets. Moreover, the comparison of the mapping between sig $C p G s$ and genes harboring sig $C p G s$ in their promoter shows that the PCHiC based annotation of $\mathrm{CpGs}$ to promoters reveals a higher overlap than Illumina annotation, Fisher's exact test $p$-value $=7.34 \mathrm{e}-08$ (Additional File 2: Table S4b), suggesting that associating $\mathrm{CpGs}$ to only the closest promoter could be limiting.

We reasoned that genes whose promoter methylation is cell-type specific should be important, even if they are not included in the gene expression signature. We therefore created an expanded gene expression deconvolution signature matrix by adding these 314 genes, that had sig CpGs in their promoters but were not sig genes, to the BPRNACan signature, leading to the BPRNACanProMet signature.

Given the importance of gene regulation by genomically distal regulatory elements that are brought into 3D contact with the promoter, having sig CpGs in both the promoter and a distal interacting fragment could strengthen the benefit of including the gene in the gene expression deconvolution signature. We therefore considered a more stringent expansion of our BPRNACan signature matrix to include only the genes that have sig $\mathrm{CpGs}$ both inside their promoter and in regions that contact their promoter in 3D (BPRNACan3DProMet).

Finally, we considered expanding our BPRNACan signature with genes that have sig CpGs only in regions that are contacting their promoters (and not directly in their promoter), giving rise to the BPRNACan3DMet signature matrix, which included a large number of genes $(\mathrm{N}=3999)$.

The principle of gene inclusion in these 3 signatures is detailed in Fig. 7. We found the BPRNACanProMet and the BPRNACan3DProMet signature matrices to have better performance compared to the original BPRNACan signature matrix. (Additional File 3: Table S6 and S7 and Additional File 2: Figure S7). On the contrary BPRNACan3DMet included too many genes and did not improve performance.

\section{Using deconvolution to predict response to immune checkpoint inhibitors}

We have so far compared performances of different deconvolution methods and signatures based on cell type composition estimates provided by FACS, H\&E image quantification of specific markers or single-cell data. All of these methods, however, carry their own biases in estimating composition. One of the main reasons that we are interested in quantifying cell types inside the TME is to better understand and predict response to immune check-point inhibitors in a personalized medicine framework. This remains challenging and biomarkers of response are needed to achieve better outcomes in immunotherapy. We therefore decided to test the pertinence of our signature matrices to produce features for models that predict response to immunotherapy based on bulk transcriptomics (Fig. 8a).

To this end, we made predictors of response to anti-PD1 antibodies using different deconvolution results as features and evaluated their performance on 3 public melanoma datasets and one bladder cancer dataset [40-43] with response to anti-PD1 through ElasticNet penalized logistic regression ${ }^{62}$ (see Methods and Fig. 8b). 
Our signature matrices in combination with Epidish or DeconRNA allowed us to train models with a performance that was better than random in a classification task (Receiving Operator Characteristic (ROC) Area Under the Curve (AUC) score above 0.5 on the standard Cross Validation (CV) training for 10 models among 12) and better than using quanTlseq or MCPCounter. On specific datasets unseen during training (see Methods) our signatures reached $A \cup C=0.77$, while training on all samples (standard $C V$ ), the BPRNACanProMet signature used by Epidish was the top performing model, with a ROC AUC score of 0.703 (Fig. 8c). The BPRNACan signature in combination with deconRNAseq is the only one that could produce models with a ROC AUC score always above 0.5 (Fig. 8c), even when predicting on datasets that had not been used for training. When excluding it from training, the Snyder bladder cancer dataset is the one with the overall worst performing models, which is probably because it is the only dataset with bladder cancer samples, all the other ones corresponding to melanoma [40-42].

Looking at the coefficients in our regression models we can estimate which variables are associated with either progressive diseases or response to therapy. The coefficients of the model trained with the combination of the BPRNACanProMet signature and the Epidish method show that B cells [57-59] (presumably indicative of the presence of tertiary lymphoid structures) and $\mathrm{M0}$ macrophages proportions are associated with patient response, whereas M2 macrophages [60], CD4, CD8 and NK cells associated with progressive disease in this model (Fig. 8d). Proportions of cancer cells, M1 macrophages, monocytes and neutrophils were not significantly associated with either of the two outcomes. We can also consider the combination of the BPRNACan signature with deconRNAseq that always produced models performing better than a random predictor, and that is the second best when the Snyder dataset was used as a test set. The coefficients of this model indicate that B cells, M0 and M1 macrophages [61] proportions are associated with response of patients, whereas CD4 and CD8 cells, Nk cells, monocytes and M2 macrophages are associated with progressive disease in this model (Fig. 8d). Like the previous model, proportions of cancer cells and neutrophils were not strongly associated with any of the two outcomes. Thus, the two models differ in the importance of M1 macrophages and monocytes for predicting response or progressive disease. 


\section{Discussion}

Numerous reference-based deconvolution methods using DNA methylation or gene expression can be used to estimate the proportion of cell types in bulk datasets from cell mixtures, such as EpiDISH, MethylCibersort (for DNAm) and CIBERSORT, MCP-counter, quanTIseq, DeconRNASeq (for GE). Moreover, there are methods that can accurately predict the proportion of cancer cells in tumor samples (purity). However, for application in immuno-oncology it can be important to estimate the proportions of cancer and specific immune cells at the same time.

Recently, Chakravarthy et al.[48] have reported that deconvolution methods based on gene expression or DNA methylation could be complementary to each other in cases where both data types are available. Despite this, they did not further explore if the reference signature matrices for each type of data can be related. Often in the clinic only gene expression data is available, so the integration of the two approaches is not common. Here we present five novel signature matrices for reference-based deconvolution:

BPmetCan: which is able to deconvolve the proportion of cancer and immune cell types from DNA methylation data (Illumina Arrays or WGBS), based on WGBS signature matrices, which we validate against MethyICIBERSORT, EpiDISH and various methods to estimate tumor purity in blood and especially in tumor samples.

CCLE_TIL10: which combines the TIL10 signature matrix [29] for immune cell types with a new list of genes identified to be cancer-cell specific using data from GTEx and CCLE, which displays excellent performance on in-vitro and in-silico mixtures of cancer cell lines and immune cells.

BPRNACan: which combines our Blueprint derived immune cell signature matrix (BPRNA) with genes that are discriminant of cancer tissues compared to normal and outperforms or equals quanTIseq and MCP-Counter on cancer samples for many of the cell types.

BPRNACanProMet: which is an enhancement of the BPRNACan signature matrix by adding genes that have a sig $C p G$ contained in their promoter, with demonstrated improvement in performance on many datasets

BPRNACan3DProMet: which is an enhancement of the BPRNACan signature matrix by adding genes that have a sig $C p G$ in their promoter and whose promoters also have a 3D contact with a fragment containing a sig $C p G$.

We performed extensive validation using previous studies, such as whole blood mixtures, solid tumor-TCGA, PBMC, multiple myeloma patient bone marrow samples and melanoma non metastatic and metastatic samples. We compared the available DNAm or GE signature matrices to demonstrate that our novel signature matrices could faithfully estimate the fraction of cancer and specific immune cell compositions from DNA methylation and bulk gene expression data. Our signature matrices can be applied to solid tumors, as confirmed by the validations presented above, but they are likely to have limited use for hematological malignancies, in which the presence of cancerous immune cells could confound the estimations, and for which more targeted signature matrices should be developed. 
We also showed that our DNAm signature matrix BPmet is more robust than others for estimating the proportions of cell types in Whole Blood samples. Moreover, our new gene expression signature matrix BPRNACan displayed higher accuracy on the predicted cell fractions on in-vivo cancer samples. Additionally, application of our GE signature matrices to publicly available data using our predicting model in this study revealed several important biological insights on predicting response to immunotherapy.

Despite the overall accuracy of our signature matrices, we found that the performance in the estimation for some cell types is lower than what we expected, probably due to the number or condition of the cell types used for establishing the reference profiles. For instance, we observed that the correlation of NK cells was lower than expected using both BPmet and BPRNA (Fig. 5a-b, and Additional file 2: Figure S1a, S2a). This may be explained as we had only $n=2$ samples to create the NK reference expression profile. Moreover, we observed a very low performance in predicting M2 macrophages in the in-silico tumor samples using BPRNACan (Additional file 2: Figure S4b). This may be explained by the fact that this M2 state in-vitro is artificially induced by cytokines to mimic the context of the TME, thereby the GE reference profiles corresponding to $\mathrm{M} 2$ cells might not capture the M2s that are found, albeit at low frequency [62] in artificial in-silico mixtures of purified cells. As opposed to NK and M2 cells, CD8 T cells and Macrophages were better predicted using GE signature matrices on cancer samples (Fig. 6d and Additional file 2: Figure S4 and S6) rather than on PBMCs (Fig. 5a). This could be explained by the differences in cell proportions and cell states between the tumor microenvironment and circulating blood. For example, only a few activated CD8 T cells and no macrophages can be found in circulating blood, but their detection in tumors is key.

We also found a low performance in detecting neutrophils according to proportions derived by H\&E (Fig. 4b, 6a). This may be explained by the fact that the reference profiles of neutrophils used to build the signature matrix are unlikely to capture all the phenotypes that neutrophils can display, especially inside tumors. Like macrophages, Tumor Associated Neutrophils (TANs) can display at least two different phenotypes - one characterised by proinflammatory programs and antitumorigenic functions and the second characterised by a protumorigenic activity $[63,64]$. Indeed neutrophils' gene expression and methylation were found to be extremely variable across individuals [65], time of the day, as well as across the different parts of the body in which they are found, highlighting their extreme plasticity $[66,67]$.

We also demonstrated that our GE signature matrices often perform better at estimating cell types proportions with EpiDISH rather than with deconRNASeq. This result is generally consistent between blood or tumor samples, except for a higher correlation of CD4 T cells while using deconRNAseq in PBMC data (Fig. 5b).

Another important novel insight of our study is that the GE signature matrix can be improved by incorporating specific genes that are associated with $\mathrm{CpGs}$ which are included in the DNA methylation signature matrix. This can be done by either identifying genes that harbor signature matrix $\mathrm{CpGs}$ (sig $\mathrm{CpGs}$ ) in their promoter, or those genes whose promoters also have a 3D contact with a fragment containing a sig $C p G$. We are thus able to expand the list 
of genes to be used in performing RNAseq-based deconvolution using information gathered from the DNA methylation signature matrix we have generated (Additional file 2: Table S2). Importantly, this expanded GE signature matrix (Additional file 2: Table S6-S7) can be applied to deconvolve samples for which only RNAseq data is available.

Despite our DNAm and RNAseq reference-based signature matrices displaying comparable to or better performance than existing methods in whole blood or cancer samples, several issues will require further investigation. We are limited by the number of samples available for specific cell types (such as NK cells) and by the fact that these profiles are generated from purified cells that are isolated from their natural environment. This is especially true for cells that acquire specific phenotypes in a TME context, such as TAMs and TANs. The availability of single-cell RNAseq datasets from cancer samples, especially from technologies that also provide protein marker quantification such as CITEseq, will greatly improve our chances of generating relevant signature matrices for any cell type of interest. For this reason we provide code for the generation of new reference signature matrices in our openly available repository.

Importantly, one of the main applications of deconvolution in immuno-oncology will be the prediction of response to immunotherapy, which can be made based on the inferred cell type proportions. We therefore benchmarked our new signature matrices and methods by evaluating their accuracy in predicting response to anti-PD1 agents in 4 public datasets. This type of exercise is aimed at identifying which signature matrices and methods uncover the presence of specific cell subtypes that can impact immune checkpoint blocker response. These important subtypes might not correspond easily to literature definitions or FACS derived populations. M2 macrophages are known to impair response to immune checkpoint blockade [60] and we confirm their association with negative prognosis despite the discrepancy between our quantification of M2s and FACS estimates. This could be due to our signature capturing specific phenotypes that are not entirely determined by traditional cell surface markers used in FACS. Interestingly, B cells and M1 macrophages have recently been proposed as potential predictors of response to immunotherapies [57-59,61]. To our knowledge, the proportion of M0 (naïve) macrophages has not been reported as predictor of response to immunotherapies, but both of our models suggest it is a potential relevant subtype in this respect. Our two models also suggest that proportions of CD4, CD8 and NK cells are associated with progressive disease, but this has to be considered with caution, as response to immunotherapy was shown to depend on the proportion of sub-cell types like memory, effector, and senescent phenotypes or defined by the presence or absence of several proteins like PD-1, PD-L1, CTLA-4, LAG-3 or TIGIT on these cells' surfaces, which is not captured by our signatures.

In summary, we have shown the potential of our gene expression signature matrices to estimate the presence of immune populations that can be predictive of the response to checkpoint blockade, bringing us closer to personalized approaches and revealing resistance mechanisms. 


\section{Conclusion}

We have presented and thoroughly validated five novel deconvolution signature matrices BPmetCan, CCLE_TIL10, BPRNACan, BPRNACanProMet and BPRNACan3DProMet, which show good performances in estimating the proportion of cell types from blood and tumor samples. Simultaneously, we have also shown that our signature matrices are more robust to estimate cell fractions compared to the other available signature matrices, and we have highlighted the relationship between genes in the expression signature matrix and $\mathrm{CpGs}$ in the methylation signature matrix. We also showed how a gene expression signature matrix can be improved by addition of genes that are recognized as being important exploiting the knowledge of $3 \mathrm{D}$ chromatin contacts between promoters and regulatory elements now available through PCHi-C networks for 17 immune cell types [21]. Our signature matrices are particularly suitable for the analysis of tumor samples from an immuno-oncology perspective, as they provide accurate estimates of immune cells as well as cancer cell proportions and have predictive power for estimating response to anti-PD1 agents. We make the signature matrices and all the code available to the community through a user-friendly snakemake pipeline that can use any given reference signature matrix to apply a variety of reference-based deconvolution methods. Additionally, the code to generate a signature matrix following the method used in this paper is also available, as well as a script to generate all the figures.

Finally, we provide a user-friendly pipeline to apply our approach and make the code available to the research community, to promote the development of new more specific deconvolution signatures (https://github.com/VeraPancaldiLab/GEMDeCan). Despite the increasing availability of single cell RNAseq data, we propose that deconvolution of cell types and subtypes from bulk transcriptomics will be a valid strategy to target immunotherapies to specific patients in a clinically relevant context and at an affordable cost.

\section{Abbreviations}

BRCA: Breast invasive carcinoma

CCLE: Cancer Cell Line Encyclopedia

DHS: DNAse hypersensitivity sites

DNAm: DNA methylation

FACS: fluorescence-activated cell sorting

FPKM: Fragments Per Kilobase of transcript per Million

GE: gene expression

GEO: Gene expression omnibus 
H\&E: Hematoxylin and eosin

IHC: Immunohistochemistry

LUAD: Lung adenocarcinoma

M: macrophages

M1: Classically activated macrophages

M2: Alternatively activated macrophages

Mono: Monocytes

Neu: Neutrophils

NK: Natural killer cells

PBMC: Peripheral blood mononuclear cells

PCHi-C: Promoter-Capture Hi-C

R: Pearson's correlation

RPC: robust partial correlation

TANs: tumor Associated Neutrophils

TAMs: tumor Associated Macrophages

TCGA: The Cancer Genome Atlas

TME: The tumor microenvironment

TPM: Transcripts per millions

Treg : Regulatory T cells

WB: whole blood

WGBS: whole-genome bisulfite sequencing

\section{Acknowledgements}

We thank members of the Pancaldi lab for critical reading of the manuscript and Sarah Djebali for help with testing the pipeline. 


\section{Funding}

This work was funded by the Chair of Bioinformatics in Oncology of the CRCT (INSERM; Fondation Toulouse Cancer Santé and Pierre Fabre Research Institute).

\section{Availability of data and materials}

The published data used is indicated in Methods. The pipeline to generate all examples is available from GitHub: https://github.com/VeraPancaldiLab/GEMDeCan and we provide an $\mathrm{R}$ notebook to reproduce all figures in the paper.

\section{Author information}

Centre de Recherches en Cancérologie de Toulouse (CRCT), INSERM U1037, Toulouse, France

Ting Xie, Julien Pernet, Nina Verstraete, Miguel Madrid Mencía, Alexis Hucteau, Alexis Coullomb, Vera Pancaldi

\section{Université Paul Sabatier III, Toulouse 31400, Toulouse, France}

Ting Xie, Julien Pernet, Nina Verstraete, Miguel Madrid Mencía, Alexis Hucteau, Alexis Coullomb, Vera Pancaldi

Translational Medicine, Institut de Recherche Pierre Fabre, Toulouse, France

Mei-Shiue Kuo, Olivier Delfour, Francisco Cruzalegui

Barcelona Supercomputing Center, Barcelona, 08034, Spain

Miguel Madrid Mencía, Vera Pancaldi

\section{Authors' contributions}

TX developed the signature matrices from the Blueprint project and performed deconvolution analyses, performed statistical analyses, and wrote the manuscript with VP. JP developed the pipeline. NV and MMM tested and corrected the pipeline, NV wrote the pipeline tutorial. MSK developed the CCLE_TIL10 signature matrix. AH contributed to improve the GE signature matrix using chromatin networks. AC predicted response to immune checkpoint inhibitors. JS revised the text and edited figures. FC, OD and VP supervised the project. All authors read and approved the final manuscript.

\section{Corresponding author}

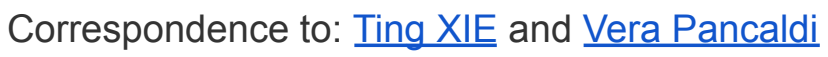

\section{Ethics approval and consent to participate}

Not applicable. 


\section{Competing interests}

The authors declare that they have no competing interests.

\section{Consent to publication}

Not Applicable.

\section{Additional files}

\section{Additional file 1:}

List of datasets used in this study for DNA methylation and gene expression reference database construction

\section{Additional file 2:}

Method S1. The threshold of log2 fold change (logFC) chosen. Figure S1. Validation of BPmet signature matrix and comparing 3 different DNA methylation signature matrices on top of 100 whole blood data with FACS (GSE132203). Figure S2. Comparing 3 different DNA methylation signature matrices in 6 WB with FACS from [33]. Figure S3. Validation of BPmetCan and comparing previously published purity estimation methods from BRCA. Figure S4. In silico validation of CCLE_TIL10 and RPRNACan from [29]. Figure S5. Comparing TCGA-LUAD tumor purity proportion using CCLE_TIL10 and BPRNACan with other previously published purity estimation methods. Figure S6. Summary of the performance of our signatures. Figure S7. Summary of the performance of our expansion gene expression signatures. Table S1. Validation datasets used to evaluate our expansion signature matrix in this study. Table S2. Main reference-based deconvolution methods used. Table S3. PCHi-C network analysis. Table S4. Fisher's test.

\section{Additional file 3:}

Seven novel deconvolution signature matrices BPmet, BPmetCan, BPRNA, BPRNACan, CCLE_TIL10, BPRNACanProMet, BPRNACan3DProMet. 


\section{References:}

1. DeBerardinis RJ. Tumor Microenvironment, Metabolism, and Immunotherapy [Internet]. New England Journal of Medicine. 2020. p. 869-71. Available from: http://dx.doi.org/10.1056/nejmcibr1914890

2. Engblom C, Pfirschke C, Pittet MJ. The role of myeloid cells in cancer therapies. Nat Rev Cancer. 2016;16:447-62.

3. O'Donnell JS, Teng MWL, Smyth MJ. Cancer immunoediting and resistance to $T$ cell-based immunotherapy [Internet]. Nature Reviews Clinical Oncology. 2019. p. 151-67. Available from: http://dx.doi.org/10.1038/s41571-018-0142-8

4. Wei SC, Duffy CR, Allison JP. Fundamental Mechanisms of Immune Checkpoint Blockade Therapy. Cancer Discov. 2018;8:1069-86.

5. Sharma P, Hu-Lieskovan S, Wargo JA, Ribas A. Primary, Adaptive, and Acquired Resistance to Cancer Immunotherapy. Cell. 2017;168:707-23.

6. Anderson KG, Stromnes IM, Greenberg PD. Obstacles Posed by the Tumor Microenvironment to T cell Activity: A Case for Synergistic Therapies [Internet]. Cancer Cell. 2017. p. 311-25. Available from: http://dx.doi.org/10.1016/j.ccell.2017.02.008

7. Mantovani A, Marchesi F, Malesci A, Laghi L, Allavena P. Tumour-associated macrophages as treatment targets in oncology. Nat Rev Clin Oncol. 2017;14:399-416.

8. Pathria P, Louis TL, Varner JA. Targeting Tumor-Associated Macrophages in Cancer [Internet]. Trends in Immunology. 2019. p. 310-27. Available from: http://dx.doi.org/10.1016/j.it.2019.02.003

9. Lawrence T, Natoli G. Transcriptional regulation of macrophage polarization: enabling diversity with identity. Nat Rev Immunol. 2011;11:750-61.

10. Murray PJ. Macrophage Polarization. Annu Rev Physiol. 2017;79:541-66.

11. Finotello F, Rieder D, Hackl H, Trajanoski Z. Next-generation computational tools for interrogating cancer immunity. Nat Rev Genet. 2019;20:724-46.

12. Aran D, Sirota M, Butte AJ. Systematic pan-cancer analysis of tumour purity. Nat Commun. 2015;6:8971.

13. Zou J, Lippert C, Heckerman D, Aryee M, Listgarten J. Epigenome-wide association studies without the need for cell-type composition. Nat Methods. 2014;11:309-11.

14. Houseman EA, Molitor J, Marsit CJ. Reference-free cell mixture adjustments in analysis of DNA methylation data. Bioinformatics. 2014;30:1431-9.

15. Houseman EA, Accomando WP, Koestler DC, Christensen BC, Marsit CJ, Nelson HH, et al. DNA methylation arrays as surrogate measures of cell mixture distribution. BMC Bioinformatics. 2012;13:86.

16. Visvader JE. Cells of origin in cancer [Internet]. Nature. 2011. p. 314-22. Available from: http://dx.doi.org/10.1038/nature09781

17. Titus AJ, Gallimore RM, Salas LA, Christensen BC. Cell-type deconvolution from DNA 
methylation: a review of recent applications. Hum Mol Genet. 2017;26:R216-24.

18. Cavalli G, Heard E. Advances in epigenetics link genetics to the environment and disease. Nature. 2019;571:489-99.

19. Timp W, Bravo HC, McDonald OG, Goggins M, Umbricht C, Zeiger M, et al. Large hypomethylated blocks as a universal defining epigenetic alteration in human solid tumors. Genome Med. 2014;6:61.

20. Stunnenberg HG, Hirst M. The International Human Epigenome Consortium: A Blueprint for Scientific Collaboration and Discovery [Internet]. Cell. 2016. p. 1897. Available from: http://dx.doi.org/10.1016/j.cell.2016.12.002

21. Javierre BM, Burren OS, Wilder SP, Kreuzhuber R, Hill SM, Sewitz S, et al. Lineage-Specific Genome Architecture Links Enhancers and Non-coding Disease Variants to Target Gene Promoters. Cell. 2016;167:1369-84.e19.

22. Brinkman AB, Nik-Zainal S, Simmer F, Rodríguez-González FG, Smid M, Alexandrov LB, et al. Partially methylated domains are hypervariable in breast cancer and fuel widespread CpG island hypermethylation. Nat Commun. 2019;10:1749.

23. Ziller MJ, Hansen KD, Meissner A, Aryee MJ. Coverage recommendations for methylation analysis by whole-genome bisulfite sequencing. Nat Methods. 2015;12:230-2, 1 p following 232.

24. Hastie T, Tibshirani R, Narasimhan B, Chu G. impute: impute: Imputation for microarray data. 2020.

25. Ritchie ME, Phipson B, Wu D, Hu Y, Law CW, Shi W, et al. limma powers differential expression analyses for RNA-sequencing and microarray studies. Nucleic Acids Res. 2015;43:e47.

26. Farha M, Jairath NK, Lawrence TS, El Naqa I. Characterization of the Tumor Immune Microenvironment Identifies MO Macrophage-Enriched Cluster as a Poor Prognostic Factor in Hepatocellular Carcinoma. JCO Clin Cancer Inform. 2020;4:1002-13.

27. Binnewies M, Roberts EW, Kersten K, Chan V, Fearon DF, Merad M, et al. Understanding the tumor immune microenvironment (TIME) for effective therapy. Nat Med. 2018;24:541-50.

28. Wang Q, Armenia J, Zhang C, Penson AV, Reznik E, Zhang L, et al. Unifying cancer and normal RNA sequencing data from different sources. Sci Data. 2018;5:180061.

29. Finotello F, Mayer C, Plattner C, Laschober G, Rieder D, Hackl H, et al. Molecular and pharmacological modulators of the tumor immune contexture revealed by deconvolution of RNA-seq data. Genome Med. 2019;11:34.

30. Ghandi M, Huang FW, Jané-Valbuena J, Kryukov GV, Lo CC, McDonald ER 3rd, et al. Next-generation characterization of the Cancer Cell Line Encyclopedia. Nature.

2019;569:503-8.

31. Carithers LJ, Moore HM. The Genotype-Tissue Expression (GTEx) Project [Internet]. Biopreservation and Biobanking. 2015. p. 307-8. Available from:

http://dx.doi.org/10.1089/bio.2015.29031.hmm 
32. Cairns J, Freire-Pritchett P, Wingett SW, Várnai C, Dimond A, Plagnol V, et al.

CHiCAGO: robust detection of DNA looping interactions in Capture Hi-C data. Genome Biol. 2016;17:127.

33. Koestler DC, Jones MJ, Usset J, Christensen BC, Butler RA, Kobor MS, et al. Improving cell mixture deconvolution by identifying optimal DNA methylation libraries (IDOL) [Internet]. BMC Bioinformatics. 2016. Available from: http://dx.doi.org/10.1186/s12859-016-0943-7

34. Teschendorff AE, Breeze CE, Zheng SC, Beck S. A comparison of reference-based algorithms for correcting cell-type heterogeneity in Epigenome-Wide Association Studies. BMC Bioinformatics. 2017;18:105.

35. Monaco G, Lee B, Xu W, Mustafah S, Hwang YY, Carré C, et al. RNA-Seq Signatures Normalized by mRNA Abundance Allow Absolute Deconvolution of Human Immune Cell Types. Cell Rep. 2019;26:1627-40.e7.

36. Racle J, de Jonge K, Baumgaertner P, Speiser DE, Gfeller D. Simultaneous enumeration of cancer and immune cell types from bulk tumor gene expression data. Elife [Internet]. 2017;6. Available from: http://dx.doi.org/10.7554/eLife.26476

37. Tirosh I, Izar B, Prakadan SM, Wadsworth MH 2nd, Treacy D, Trombetta JJ, et al. Dissecting the multicellular ecosystem of metastatic melanoma by single-cell RNA-seq. Science. 2016;352:189-96.

38. Samur MK. RTCGAToolbox: a new tool for exporting TCGA Firehose data. PLoS One. 2014;9:e106397.

39. Nakamura K, Kassem S, Cleynen A, Chrétien M-L, Guillerey C, Putz EM, et al. Dysregulated IL-18 Is a Key Driver of Immunosuppression and a Possible Therapeutic Target in the Multiple Myeloma Microenvironment [Internet]. Cancer Cell. 2018. p. 634-48.e5. Available from: http://dx.doi.org/10.1016/j.ccell.2018.02.007

40. Hugo W, Zaretsky JM, Sun L, Song C, Moreno BH, Hu-Lieskovan S, et al. Genomic and Transcriptomic Features of Response to Anti-PD-1 Therapy in Metastatic Melanoma. Cell. 2017;168:542.

41. Riaz N, Havel JJ, Makarov V, Desrichard A, Urba WJ, Sims JS, et al. Tumor and Microenvironment Evolution during Immunotherapy with Nivolumab. Cell. 2017;171:934-49.e16.

42. Gide TN, Quek C, Menzies AM, Tasker AT, Shang P, Holst J, et al. Distinct Immune Cell Populations Define Response to Anti-PD-1 Monotherapy and Anti-PD-1/Anti-CTLA-4 Combined Therapy. Cancer Cell. 2019;35:238-55.e6.

43. Snyder A, Nathanson T, Funt SA, Ahuja A, Buros Novik J, Hellmann MD, et al. Contribution of systemic and somatic factors to clinical response and resistance to PD-L1 blockade in urothelial cancer: An exploratory multi-omic analysis. PLoS Med. 2017; 14:e1002309.

44. Friedman J, Hastie T, Tibshirani R. Regularization Paths for Generalized Linear Models via Coordinate Descent. J Stat Softw. 2010;33:1-22.

45. Köster J, Rahmann S. Snakemake-a scalable bioinformatics workflow engine. 
Bioinformatics. 2018;34:3600.

46. Anaconda Software Distribution. Computer software. Vers. 2-2.4.0. Anaconda, Nov. 2016. Web. .

47. Farlik M, Halbritter F, Müller F, Choudry FA, Ebert P, Klughammer J, et al. DNA Methylation Dynamics of Human Hematopoietic Stem Cell Differentiation. Cell Stem Cell. 2016;19:808-22.

48. Chakravarthy A, Furness A, Joshi K, Ghorani E, Ford K, Ward MJ, et al. Author Correction: Pan-cancer deconvolution of tumour composition using DNA methylation. Nat Commun. 2018;9:4642.

49. Thurman RE, Rynes E, Humbert R, Vierstra J, Maurano MT, Haugen E, et al. The accessible chromatin landscape of the human genome. Nature. 2012;489:75-82.

50. Carter SL, Cibulskis K, Helman E, McKenna A, Shen H, Zack T, et al. Absolute quantification of somatic DNA alterations in human cancer [Internet]. Nature Biotechnology. 2012. p. 413-21. Available from: http://dx.doi.org/10.1038/nbt.2203

51. Yoshihara K, Shahmoradgoli M, Martínez E, Vegesna R, Kim H, Torres-Garcia W, et al. Inferring tumour purity and stromal and immune cell admixture from expression data. Nat Commun. 2013;4:2612.

52. Saltz J, Gupta R, Hou L, Kurc T, Singh P, Nguyen V, et al. Spatial Organization and Molecular Correlation of Tumor-Infiltrating Lymphocytes Using Deep Learning on Pathology Images. Cell Rep. 2018;23:181-93.e7.

53. Gong T, Szustakowski JD. DeconRNASeq: a statistical framework for deconvolution of heterogeneous tissue samples based on mRNA-Seq data. Bioinformatics. 2013;29:1083-5.

54. Becht E, Giraldo NA, Lacroix L, Buttard B, Elarouci N, Petitprez F, et al. Estimating the population abundance of tissue-infiltrating immune and stromal cell populations using gene expression. Genome Biol. 2016;17:218.

55. Madrid-Mencía M, Raineri E, Cao TBN, Pancaldi V. Using GARDEN-NET and ChAseR to explore human haematopoietic 3D chromatin interaction networks. Nucleic Acids Res. 2020;48:4066-80.

56. Schoenfelder S, Furlan-Magaril M, Mifsud B, Tavares-Cadete F, Sugar R, Javierre B-M, et al. The pluripotent regulatory circuitry connecting promoters to their long-range interacting elements. Genome Res. 2015;25:582-97.

57. Petitprez F, de Reyniès A, Keung EZ, Chen TW-W, Sun C-M, Calderaro J, et al. B cells are associated with survival and immunotherapy response in sarcoma. Nature. 2020;577:556-60.

58. Cabrita R, Lauss M, Sanna A, Donia M, Skaarup Larsen M, Mitra S, et al. Tertiary lymphoid structures improve immunotherapy and survival in melanoma. Nature. 2020;577:561-5.

59. Helmink BA, Reddy SM, Gao J, Zhang S, Basar R, Thakur R, et al. B cells and tertiary lymphoid structures promote immunotherapy response. Nature. 2020;577:549-55.

60. Ceci C, Atzori MG, Lacal PM, Graziani G. Targeting Tumor-Associated Macrophages to 
Increase the Efficacy of Immune Checkpoint Inhibitors: A Glimpse into Novel Therapeutic Approaches for Metastatic Melanoma. Cancers [Internet]. 2020;12. Available from: http://dx.doi.org/10.3390/cancers12113401

61. Zeng D, Ye Z, Wu J, Zhou R, Fan X, Wang G, et al. Macrophage correlates with immunophenotype and predicts anti-PD-L1 response of urothelial cancer. Theranostics. 2020;10:7002-14.

62. Shen-Orr SS, Gaujoux R. Computational deconvolution: extracting cell type-specific information from heterogeneous samples. Curr Opin Immunol. 2013;25:571-8.

63. Shaul ME, Fridlender ZG. Tumour-associated neutrophils in patients with cancer. Nat Rev Clin Oncol. 2019;16:601-20.

64. Teijeira Á, Garasa S, Gato M, Alfaro C, Migueliz I, Cirella A, et al. CXCR1 and CXCR2 Chemokine Receptor Agonists Produced by Tumors Induce Neutrophil Extracellular Traps that Interfere with Immune Cytotoxicity. Immunity. 2020;52:856-71.e8.

65. Ecker S, Chen L, Pancaldi V, Bagger FO, Fernández JM, Carrillo de Santa Pau E, et al. Genome-wide analysis of differential transcriptional and epigenetic variability across human immune cell types. Genome Biol. 2017;18:18.

66. Giese MA, Hind LE, Huttenlocher A. Neutrophil plasticity in the tumor microenvironment. Blood. 2019;133:2159-67.

67. Sagiv JY, Michaeli J, Assi S, Mishalian I, Kisos H, Levy L, et al. Phenotypic diversity and plasticity in circulating neutrophil subpopulations in cancer. Cell Rep. 2015;10:562-73. 


\section{FIGURE LEGENDS}

a

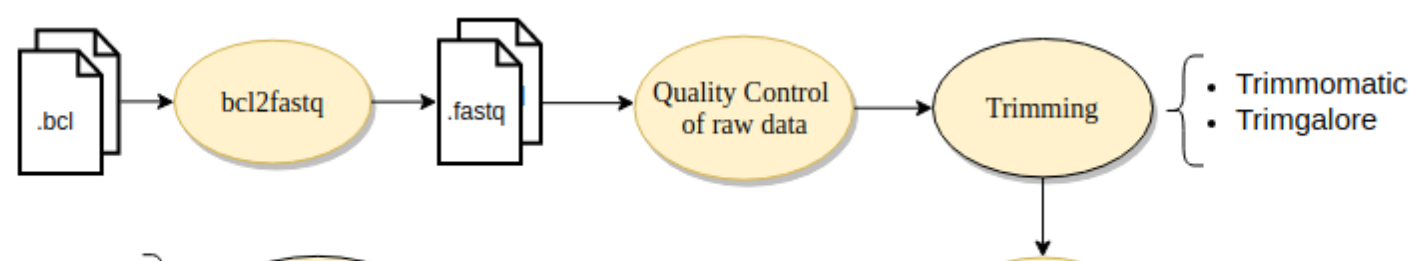

- Salmon

- Kallisto

- STAR + RSEM

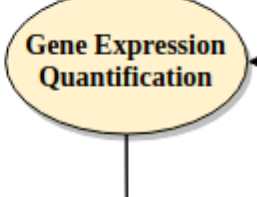

Quality Control

building

after trimming
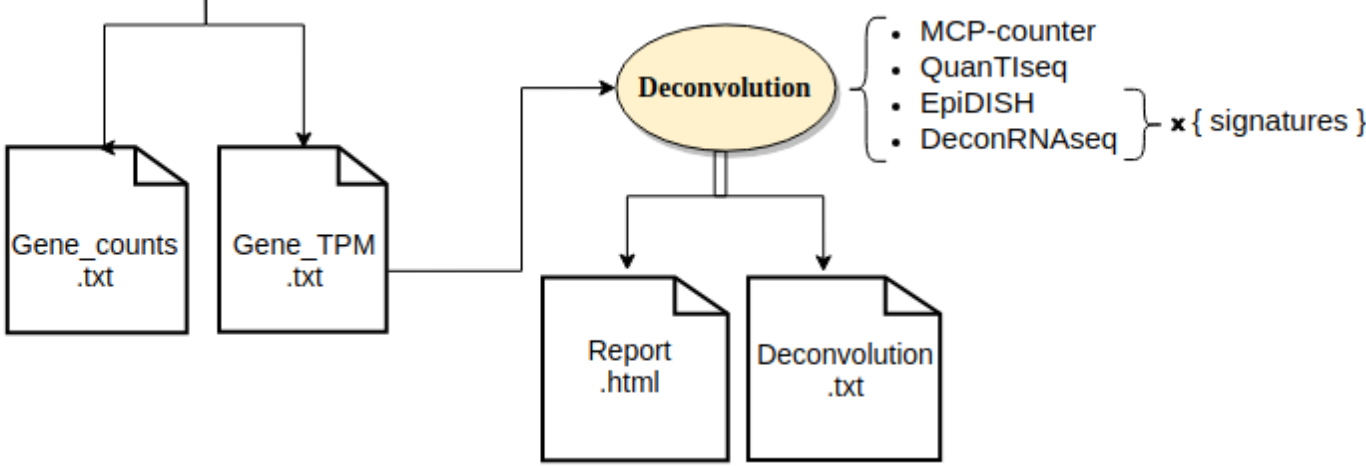

b

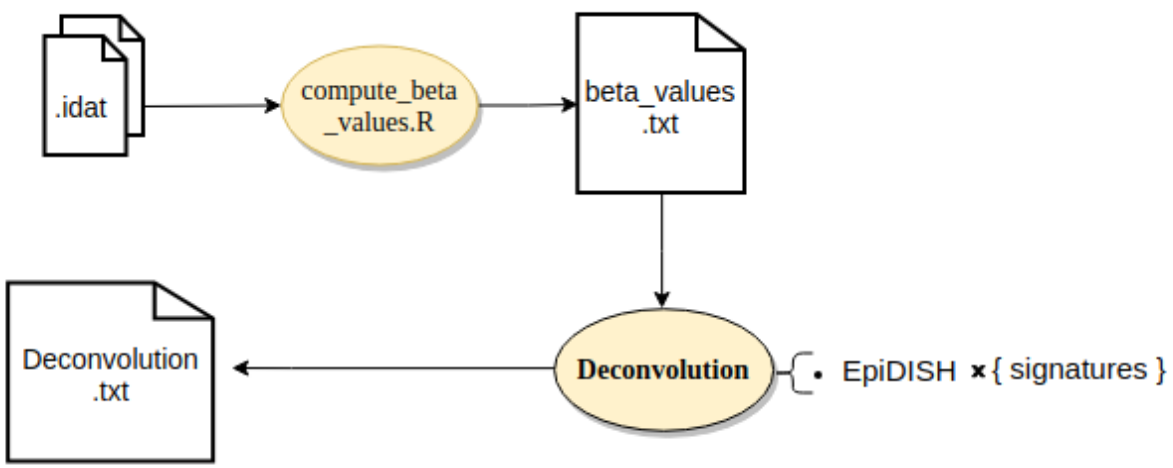

Fig. 1: Deconvolution pipeline workflow. a) RNAseq processing and deconvolution pipeline. b) DNA methylation deconvolution workflow 
a

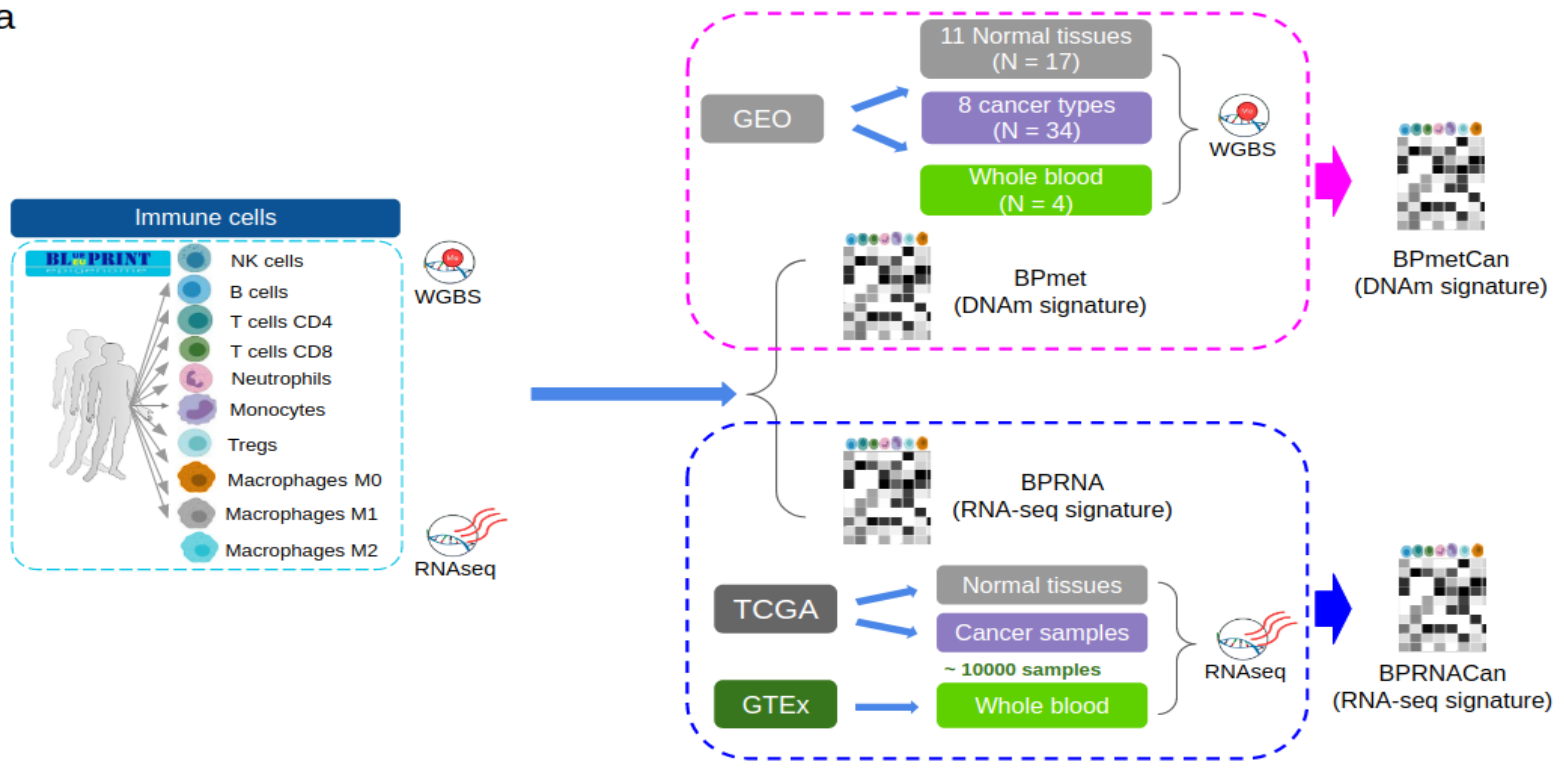

b

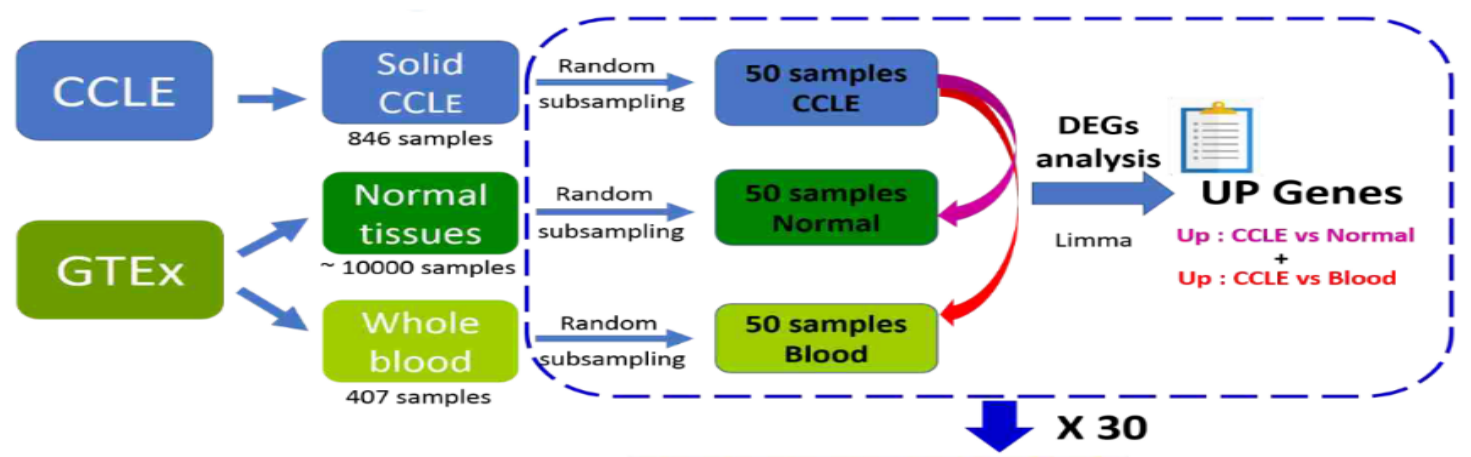

CCLE : Cancer Cell Line Encyclopedia GTEx : Genotype-Tissue Expression

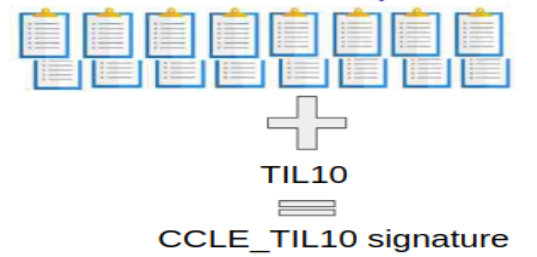

30 lists of UP Genes

C

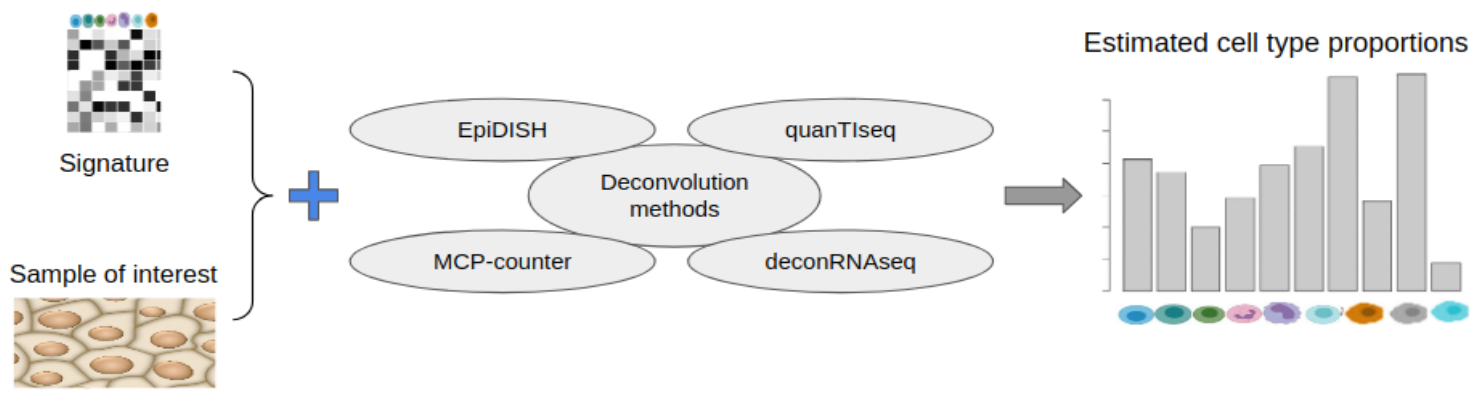

Fig. 2: Schematic description of the proposed deconvolution approach. (a) Workflow to generate BPmetCan and BPRNACan signature matrices, through combining BPmet and BPRNA immune signature matrices, and cancer signature genes and CpGs. (b) To deconvolve the exact proportion of cancer cells as well as immune cells, the TIL10 signature matrix [29] was combined with a list of genes that differs between cancer cell lines, normal tissues and whole blood, generating the CCLE_TIL10 signature matrix. (c) Using these signature matrices with deconvolution methods (based on DNAm or GE) we can estimate cancer and immune cell type proportion from bulk samples. 

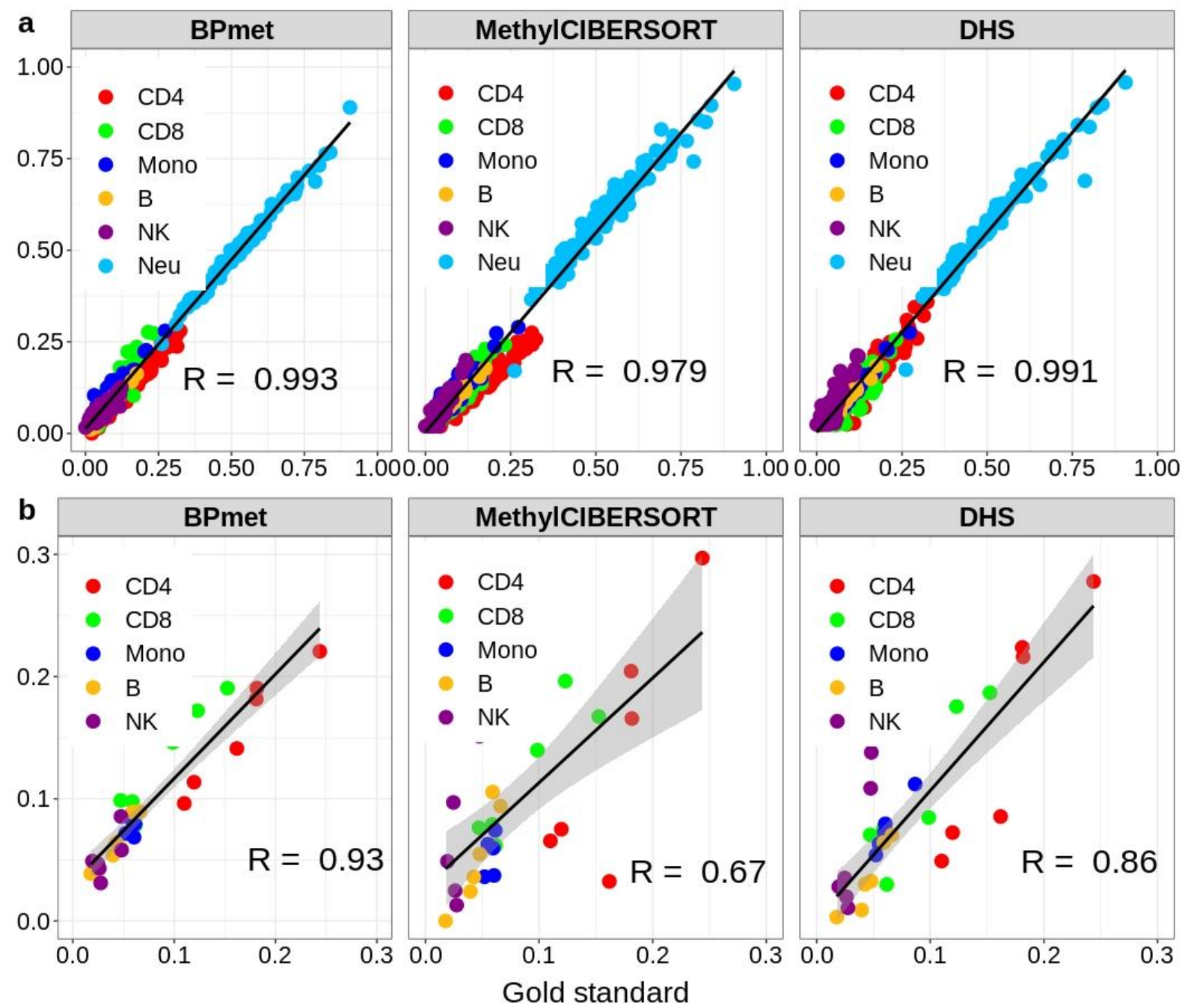

Fig. 3: Correlation of estimated cell fractions using the 3 signatures for EpiDISH vs cell fractions estimated by FACS (gold standard) (a) Comparing our DNA methylation signature matrix BPmet and available signature matrices (MethylCIBERSORT, EpiDISH-DHS) in 100 whole blood data with FACS data (GSE132203) and DNA Methylation data (Illumina EPIC 850k) from the Grady Trauma Project using the EpiDISH RPC method. (b) Comparing the DNA methylation signature matrices in 6 WB data analysed by Illumina 450k platform and by FACS (GSE77797) [33]. 

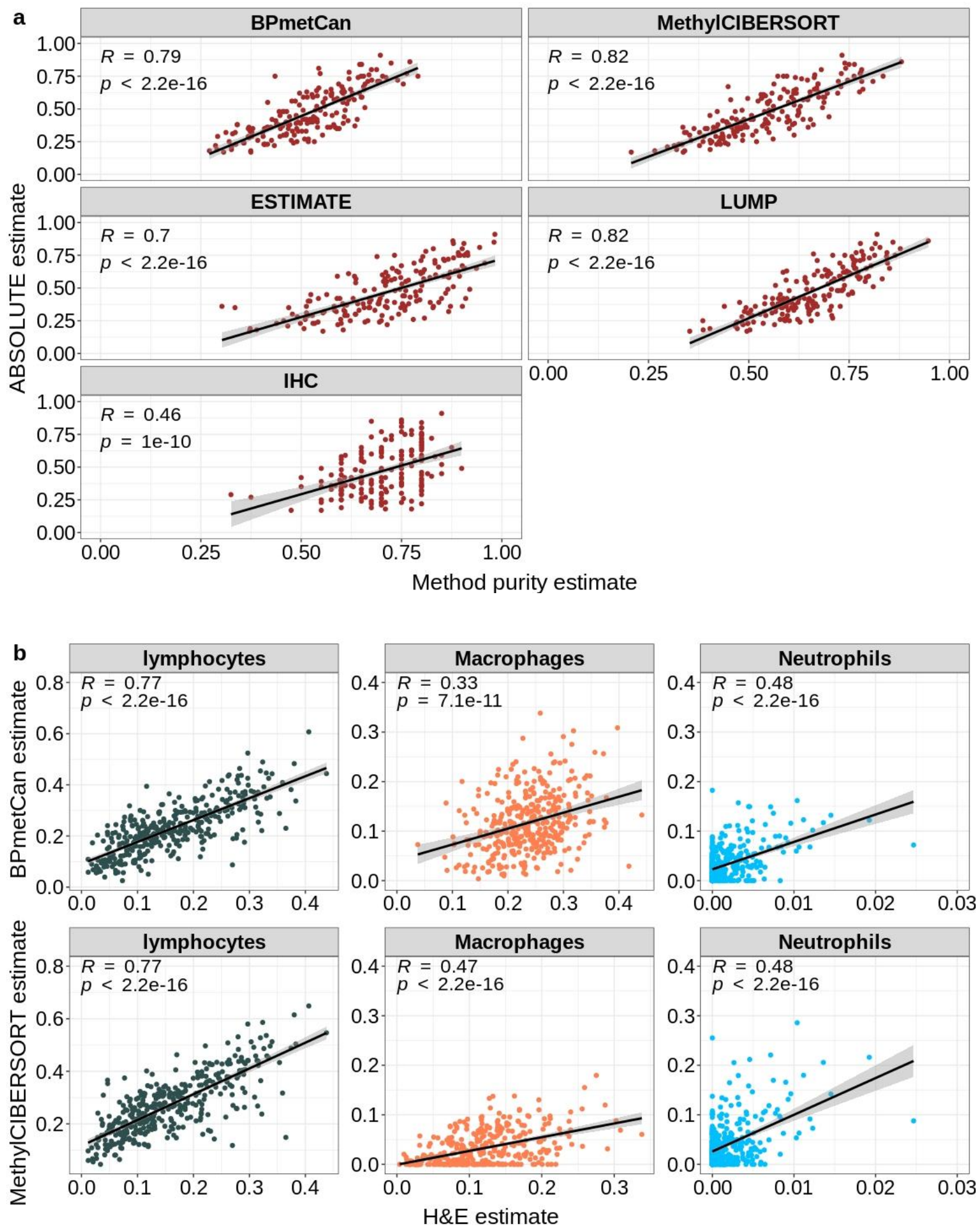

Fig. 4: Deconvolution using different DNA methylation signature matrices in tumor samples. (a) Correlations between TCGA-LUAD tumor purity proportion estimated by BPmetCan and other previously published purity estimation methods compared to estimates by ABSOLUTE using Pearson correlations [12]. (b) Scatterplots showing correlation between estimates of proportions of different immune cell types and H\&E image estimates from [52]. 


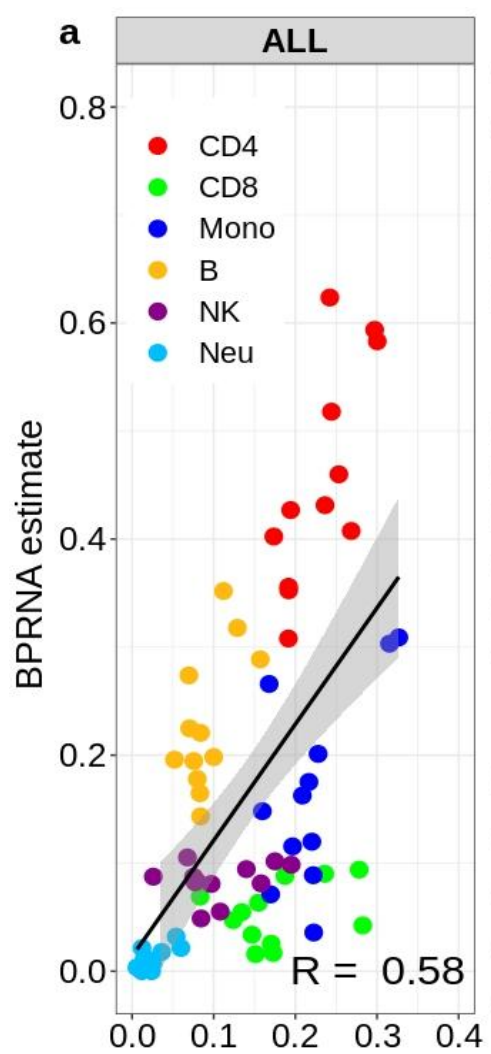

b

Pearson correlation

\begin{tabular}{|c|c|c|c|c|c|}
\hline Neutrophils & $\begin{array}{c}0.74 \\
\star \star\end{array}$ & 0.53 & -0.12 & $\begin{array}{r}0.7 \\
\star\end{array}$ & \multirow{7}{*}{$\begin{array}{l}\text { Correlation } \\
\begin{array}{l}0.75 \\
0.50 \\
0.25 \\
0.00\end{array}\end{array}$} \\
\hline NK_cells & $\begin{array}{c}0.91 \\
\star \star \star\end{array}$ & $\begin{array}{c}0.62 \\
*\end{array}$ & -0.1 & 0.25 & \\
\hline B_cells & 0.24 & $\begin{array}{l}0.9 \\
\star \star \star\end{array}$ & 0.36 & $\begin{array}{c}0.6 \\
\star\end{array}$ & \\
\hline Monocytes & $\begin{array}{c}0.89 \\
\star \star \star\end{array}$ & $\begin{array}{c}0.92 \\
\star \star \star\end{array}$ & -0.002 & $\begin{array}{c}0.58 \\
\star\end{array}$ & \\
\hline CD8 & 0.72 & $\begin{array}{c}0.82 \\
\star \star\end{array}$ & 0.31 & 0.32 & \\
\hline \multirow[t]{2}{*}{ CD4 } & NA & $\begin{array}{c}0.63 \\
\star \star\end{array}$ & $\begin{array}{c}0.79 \\
\star \star\end{array}$ & $\begin{array}{c}0.75 \\
\star \star\end{array}$ & \\
\hline & 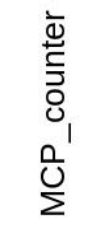 & $\begin{array}{l}\text { סू } \\
\frac{\mathscr{D}}{E} \\
\frac{\tilde{\sigma}}{\sigma} \\
\frac{\sigma}{\sigma}\end{array}$ & 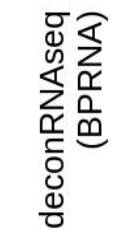 & 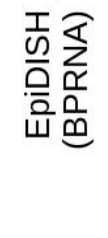 & \\
\hline
\end{tabular}

Fig. 5: Deconvolution using (a) BPRNA signature matrices and (b) Pearson Correlation between the cell fractions estimated by each method Predicted vs. FACS proportions in PBMC (GSE107011) [35]. The significance of the Pearson Correlation is indicated by stars: ${ }^{*} p<0.05,{ }^{* *} p<0.01,{ }^{* * *} p<0.001$ 

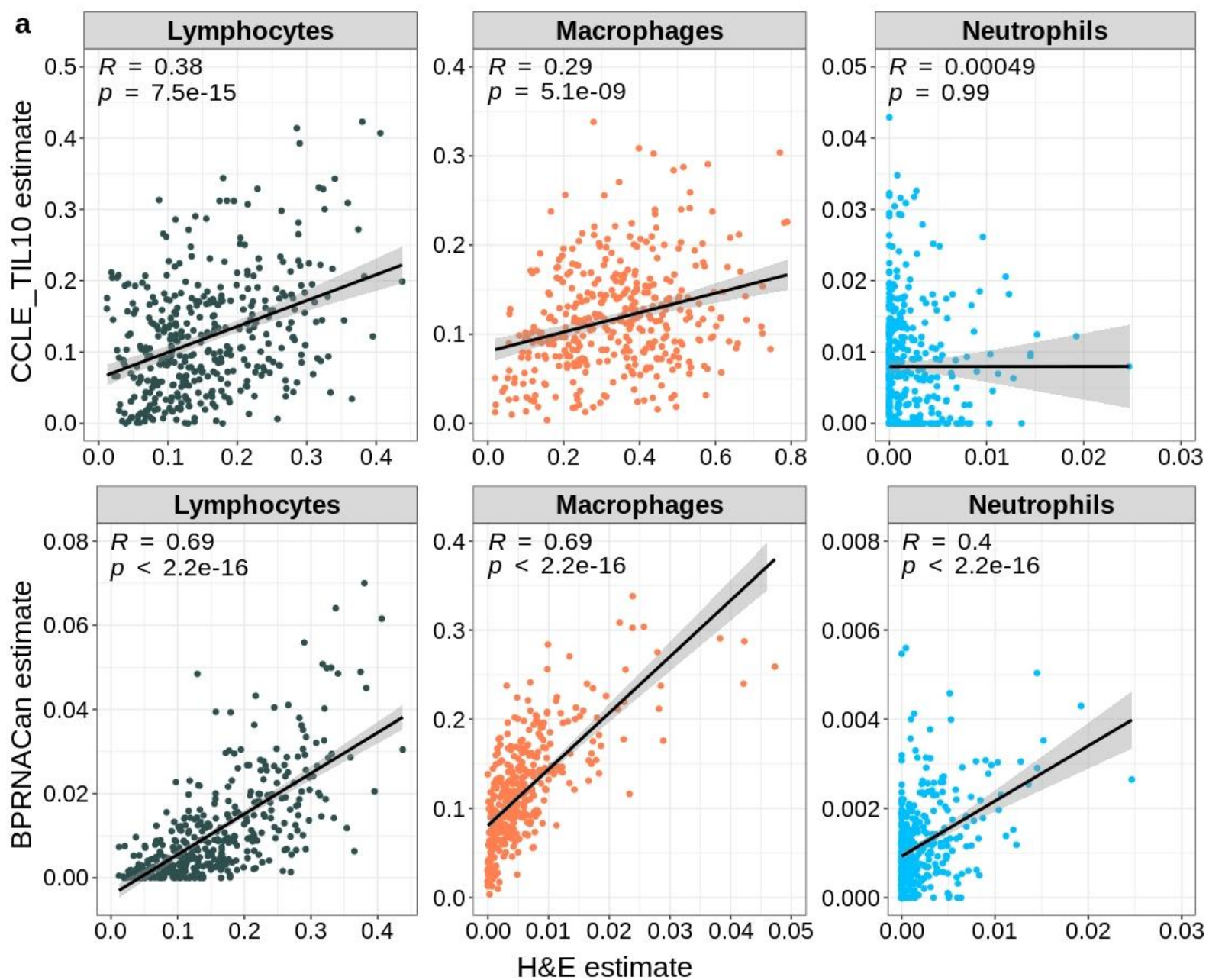

b

Pearson correlation

\begin{tabular}{|c|c|c|c|c|c|c|c|}
\hline All & NA & $\begin{array}{c}0.46 \\
\star \star \star\end{array}$ & $\begin{array}{c}0.12 \\
*\end{array}$ & $\begin{array}{c}0.18 \\
\star \star\end{array}$ & $\begin{array}{c}-0.19 \\
\star \star\end{array}$ & $\underset{*}{0.68}$ & \multirow{5}{*}{$\begin{array}{c}\text { Correlation } \\
\begin{array}{c}0.50 \\
0.25 \\
0.00\end{array}\end{array}$} \\
\hline Neutrophils & 0.06 & $\underset{* \star}{0.15}$ & -0.06 & 0.00049 & $\underset{* \star}{0.02}$ & $\begin{array}{c}0.4 \\
\star \star \star\end{array}$ & \\
\hline Macrophages & $\begin{array}{c}0.64 \\
\star \star \star\end{array}$ & $\underset{* \star * \star}{0.45}$ & $\underset{\star \star \star .54}{0.54}$ & $\begin{array}{c}0.29 \\
\star \star \star\end{array}$ & $\underset{* \star}{0.14}$ & $\begin{array}{c}0.69 \\
\star \star \star \star\end{array}$ & \\
\hline \multirow[t]{2}{*}{ Lymphocytes } & $\begin{array}{l}0.7 \\
\star \star \star\end{array}$ & $\begin{array}{l}0.7 \\
\text { ** }\end{array}$ & -0.09 & $\begin{array}{c}0.38 \\
\star \star \star\end{array}$ & -0.03 & 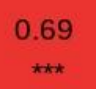 & \\
\hline & 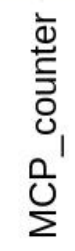 & 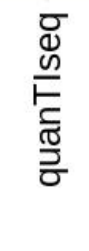 & 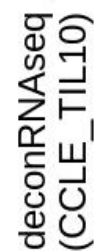 & 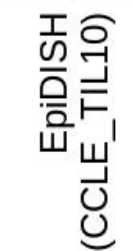 & 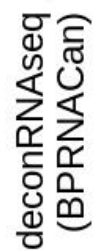 & 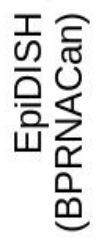 & \\
\hline
\end{tabular}



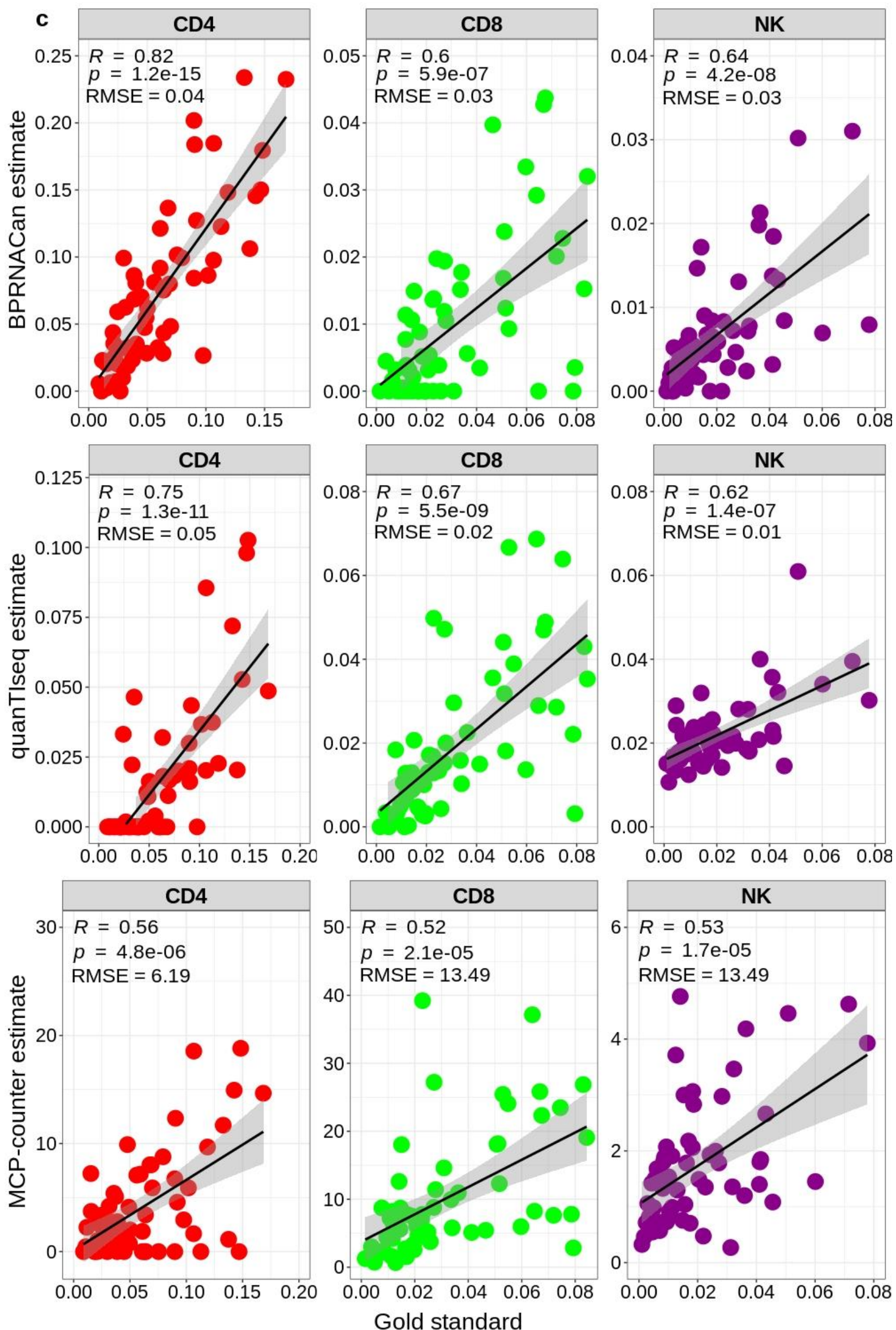

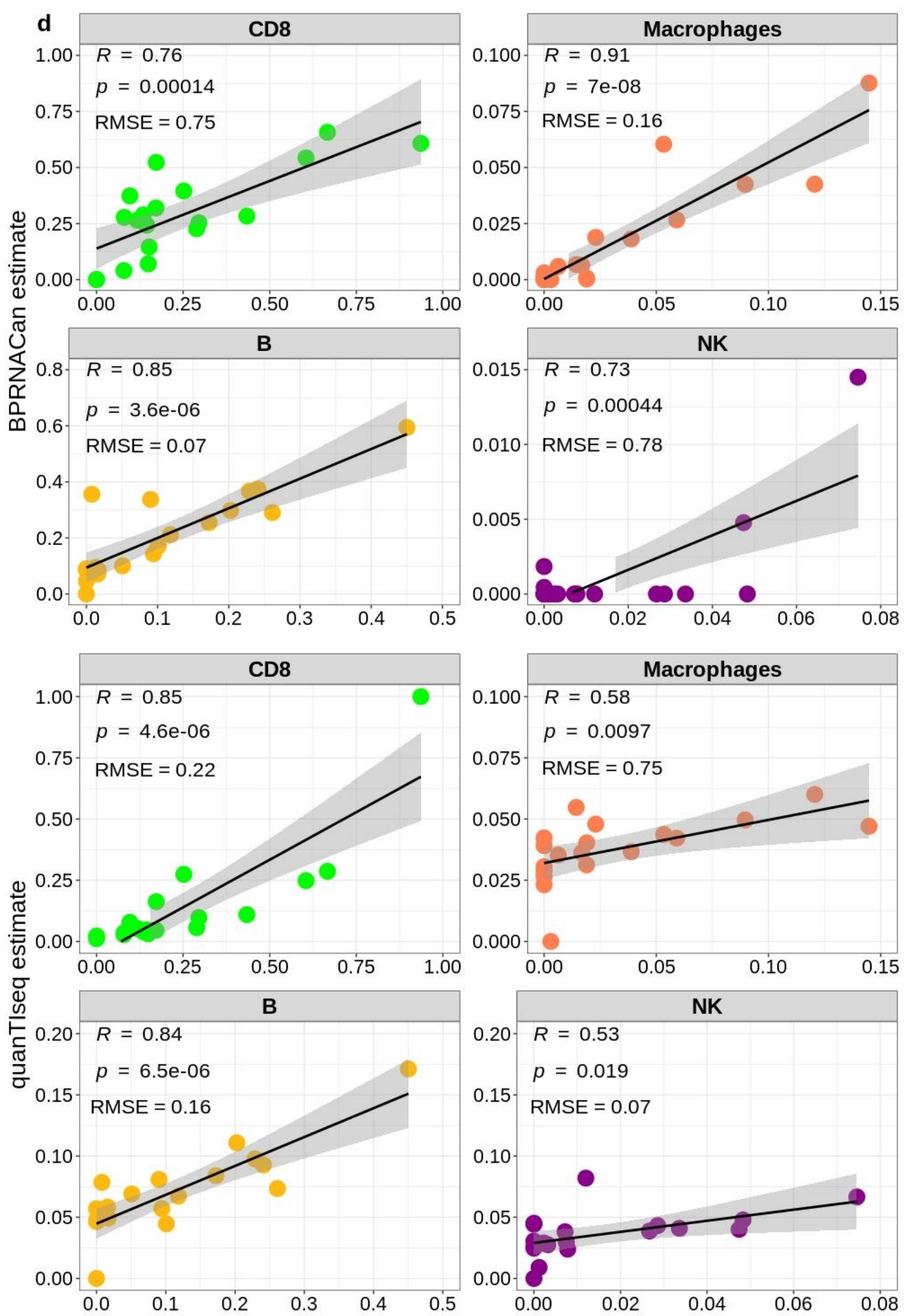

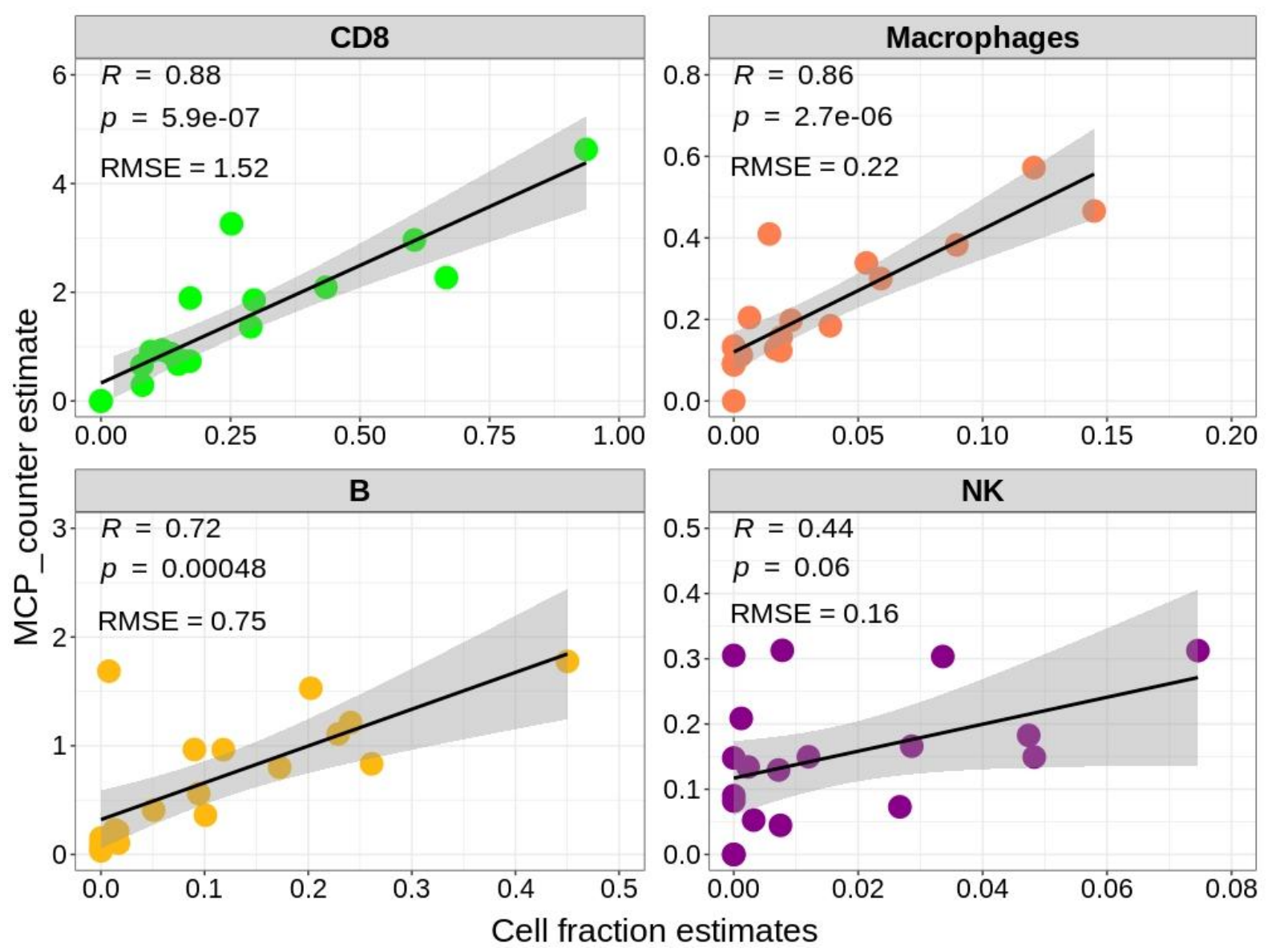

Fig. 6: Deconvolution in tumor samples (a) Comparison of the proportion of immune between CCLE_TIL10 (Top) and BPRNAcan (Bottom) and H\&E staining images in TCGA-LUAD [52]. (b) The correlation with cell type proportions measured on different datasets with samples containing cancer cells by different RNAseq reference-based deconvolution methods and experimental estimates based on H\&E analysis [52]. The significance of the Pearson Correlation is indicated by stars: ${ }^{*} p<0.05,{ }^{* *} p<0.01,{ }^{* * *} p<0.001$. (c) Comparing cell type proportions against FACS data in 59 bone marrow samples [39]. (d) Scatter plots and Pearson correlations of each cell subtype proportion predicted by the BPRNACan, quanTIseq and MCP-counter and the true cell fractions from scRNAseq data from melanoma samples [37]. 


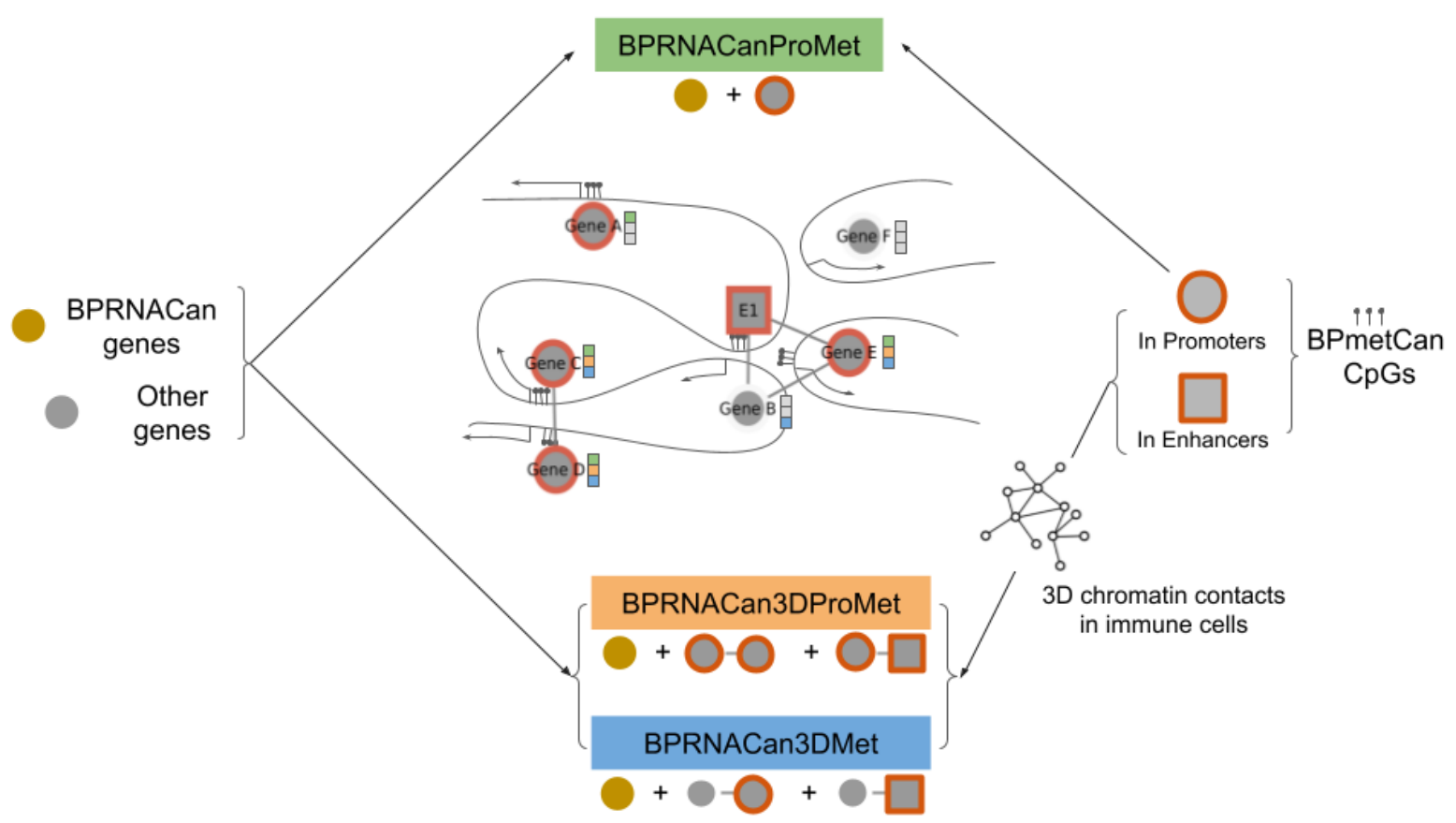

Fig. 7: Strategy of integration of BPRNACan and BPmetCan signature matrices via 3D chromatin contact networks. The BPRNACan signature is expanded including genes that have sig CpGs in their promoters (BPRNACanProMet), genes that have sig CpGs only in 3D regions in contact with their promoters (BPRNACan3DMet) and genes that have sig CpGs in both promoter and 3D contacting regions (BPRNACan3DProMet). 
bioRxiv preprint doi: https://doi.org/10.1101/2021.04.09.439207; this version posted May 4, 2021. The copyright holder for this preprint (which was not certified by peer review) is the author/funder, who has granted bioRxiv a license to display the preprint in perpetuity. It is made available under aCC-BY-NC 4.0 International license.

a

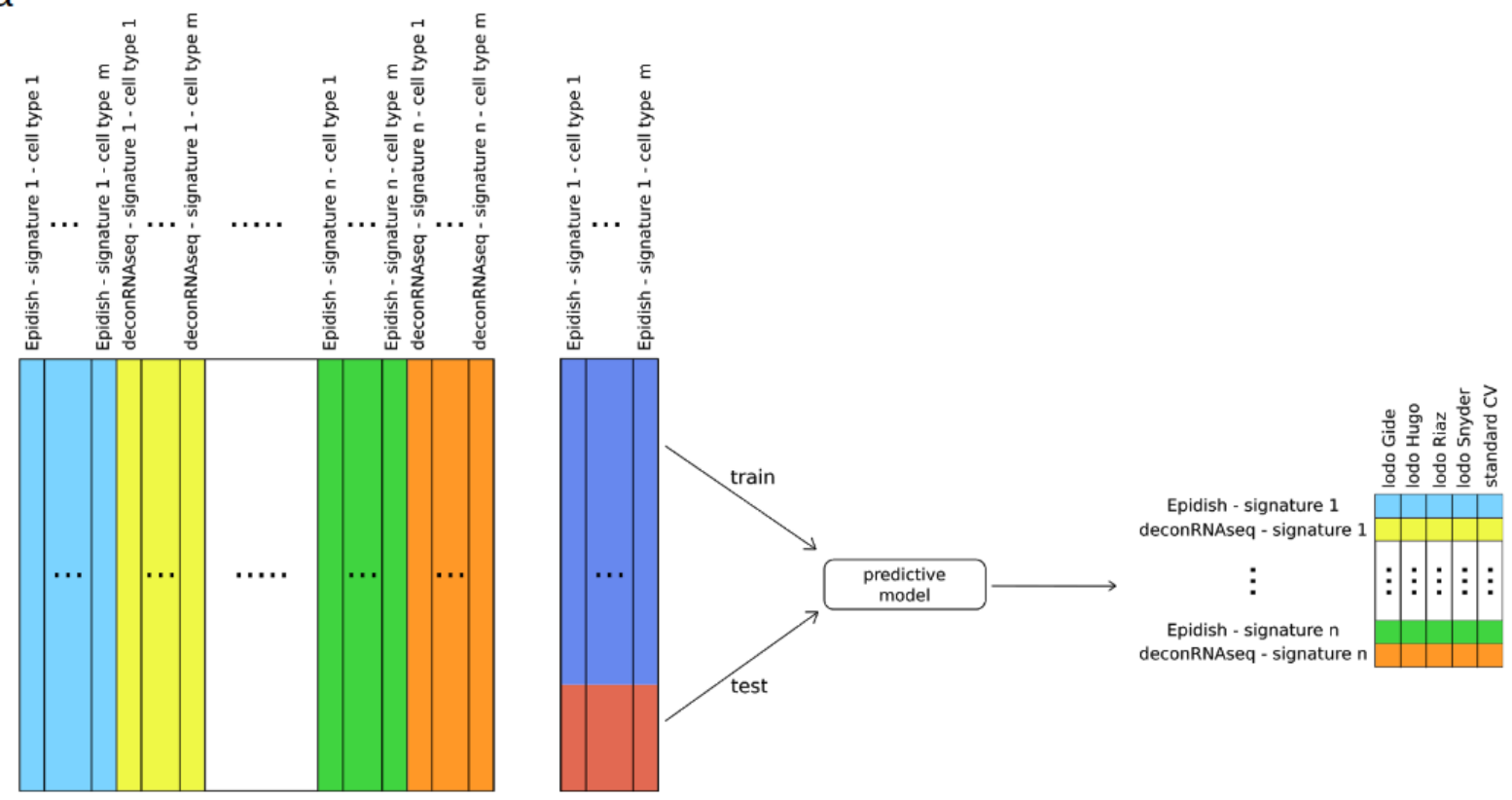

b

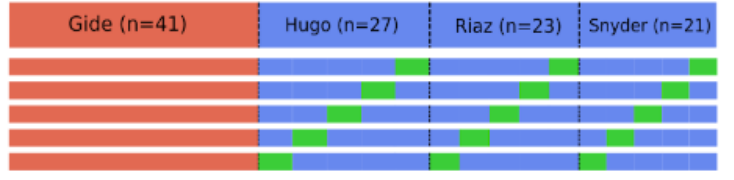

\begin{tabular}{l|l|l|l|} 
Gide $(n=41)$ & Hugo $(n=27)$ & Riaz $(n=23)$ & Snyder $(n=21)$
\end{tabular}

\begin{tabular}{l|l|l|l} 
Gide $(n=41)$ & Hugo $(n=27)$ & Riaz $(n=23)$ & Snyder $(n=21)$
\end{tabular}

Gide $(n=41)$

\begin{tabular}{l|l|l} 
Hugo $(\mathrm{n}=27)$ & Riaz $(\mathrm{n}=23)$ & Snyder $(\mathrm{n}=21)$
\end{tabular}

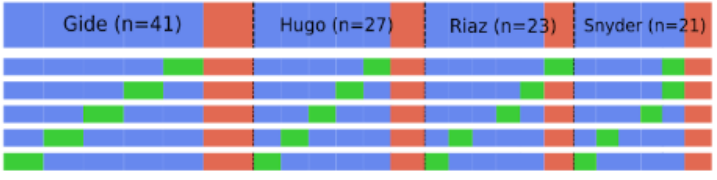

train set

validation set

test set

C

\begin{tabular}{|l|c|c|c|c|c|}
\hline Signature & Gide & Hugo & Riaz & Snyder & CV all datasets \\
\hline quanTIseq & 0.643 & 0.500 & 0.568 & 0.333 & 0.510 \\
\hline MCP-counter & 0.675 & 0.467 & 0.477 & 0.639 & 0.552 \\
\hline Epidish_BPRNACan & 0.500 & 0.478 & 0.773 & 0.491 & 0.646 \\
\hline deconRNAseq_BPRNACan & 0.655 & 0.550 & 0.667 & 0.509 & 0.594 \\
\hline Epidish_BPRNACanProMet & 0.663 & 0.606 & 0.629 & 0.472 & 0.703 \\
\hline deconRNAseq_BPRNACanProMet & 0.620 & 0.617 & 0.606 & 0.481 & 0.568 \\
\hline Epidish_BPRNACan3DProMet & 0.575 & 0.550 & 0.652 & 0.500 & 0.688 \\
\hline deconRNAseq_BPRNACan3DProMet & 0.645 & 0.606 & 0.606 & 0.463 & 0.568 \\
\hline Epidish_BPRNACan3DMet & 0.500 & 0.544 & 0.773 & 0.426 & 0.490 \\
\hline deconRNAseq_BPRNACan3DMet & 0.500 & 0.567 & 0.659 & 0.426 & 0.427 \\
\hline
\end{tabular}


d
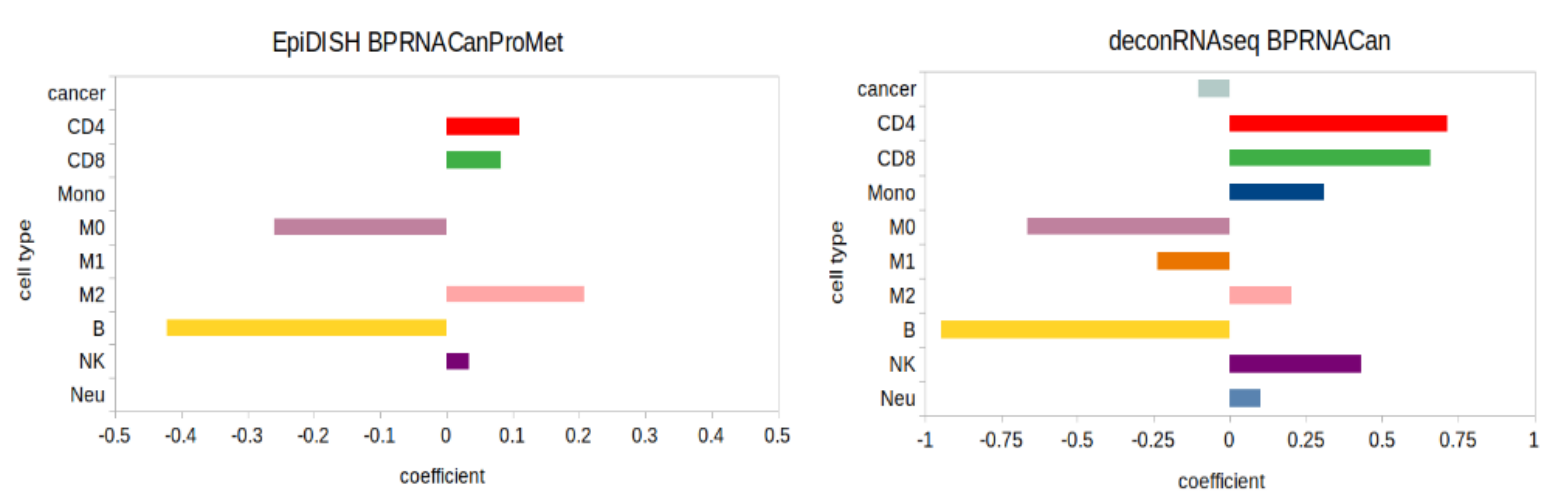

Fig. 8: Evaluation of predictive power of signatures in combinations with deconvolution methods. (a) Each combination of signature and deconvolution method was used alone to estimate cell types proportions for all samples. This data was used to train and test an elasticnet [44] penalized logistic regression model to predict response to immunotherapy. Performance of models can then be compared across signatures and training methods. (b) Left: One training method is leave-one-dataset-out (lodo), where one dataset is used for testing (red) and the other ones are used for training (blue). During each training phase, hyperparameters for the $\mathrm{I} 1$ ratio and regularization strength were searched for by 5 -fold cross validation (CV), where the training set is subdivided in 5 parts, each one of them being used as a validation set (green) while the other ones are used for training. At the end of the CV search the model is re-trained on all training samples, and tested on the test set. Right: The last training method is standard cross validation, during which a fourth of all samples is held-out as a test set. The remaining samples are used for training, with a 5-fold CV search for the hyperparameters that includes successive training sets (blue) and validation sets (green). (c) Table of models performances by combinations of signatures and deconvolution methods and by training methods. The performance is assessed with the ROC AUC score computed on test sets, which is indicated by the name of the dataset for the lodo training. For the standard CV, a fifth of samples was held out as a test set. (d) Coefficients of logistic regression models for the BPRNACanProMet signature with Epidish (left) and for the BPRNACan signature with deconRNAseq (right). Variables with negative coefficients are associated with patient response, the amplitude of coefficients is related to variable importance. 